KMU FORSCHUNG AUSTRIA

Austrian Institute for SME Research

\title{
Evaluierung der Auswirkungen der Businessforen des WIFI auf die österreichische Wirtschaft
}


Diese Studie wurde im Auftrag des Wirtschaftsförderungsinstituts (WIFI) der Wirtschaftskammer Österreich durchgeführt.

KMU FORSCHUNG AUSTRIA

Austrian Institute for SME Research

(Präsident: o. Univ.-Prof. Dr.Dr.h.c. J. Hanns Pichler, M.Sc.)

Für den Inhalt verantwortlich: Walter Bornett

Verfasser des Berichts:

Alfred Radauer

Sonja Sheikh

Layout:

Susanne Fröhlich

Dieses Werk ist urheberrechtlich geschützt. Jede Art von Nachdruck, Vervielfältigung, Übersetzung oder Einspeicherung und Verwendung in Datenverarbeitungssystemen ist ohne ausdrückliche schriftliche Zustimmung der KMU FORSCHUNG AUSTRIA verboten.

Alle Rechte, insbesondere die Rechte der Verbreitung, der Vervielfältigung, der Übersetzung, des Nachdrucks und die Wiedergabe auf fotomechanischem oder ähnlichem Wege, durch Fotokopie, Mikrofilm oder andere elektronische Verfahren sowie die Speicherung in Datenverarbeitungsanlagen, bleiben, auch bei nur auszugsweiser Verwertung, der KMU FORSCHUNG AUSTRIA vorbehalten. 


\section{Inhaltsverzeichnis}

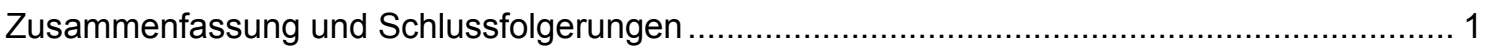

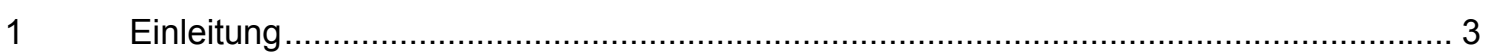

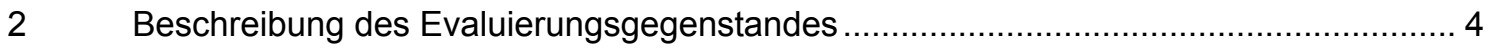

2.1 Zielsetzungen und organisatorischer Aufbau des Programms .................................... 4

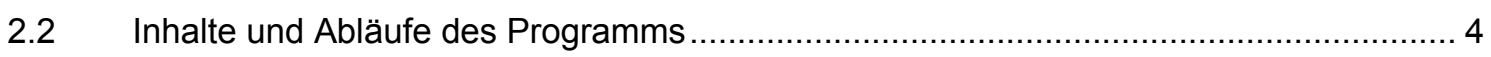

$2.3 \quad$ Der bisherige Programmverlauf in Zahlen ............................................................ 6

$3 \quad$ Methodische Vorgehensweise und Kenngrößen der empirischen Erhebung................ 10

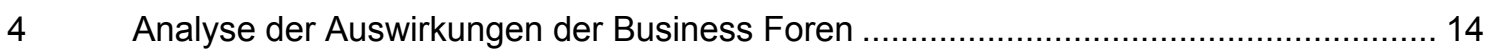

4.1 Bisherige Auslandserfahrungen der teilnehmenden Unternehmen ............................. 14

4.2 Teilnahmemotive an den Business Foren des WIFI ................................................ 16

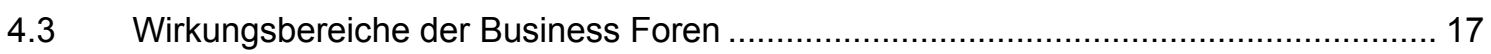

4.3.1 Kontakte zwischen österreichischen und osteuropäischen Unternehmen .................... 17

4.3.2 Vertragsabschlüsse zwischen österreichischen und osteuropäischen Unternehmen ... 19

4.3.3 Nutzen und Problembereiche für alle beteiligten Unternehmen .................................. 21

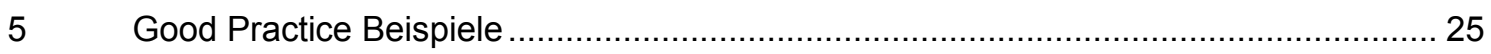

5.1 Der Dachstein- und Dachsystemhersteller BRAMAC …......................................... 25

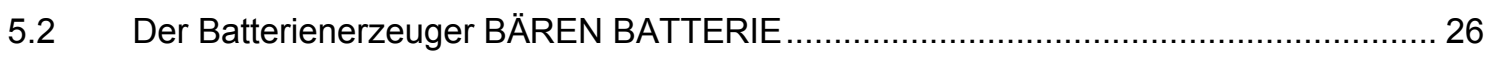

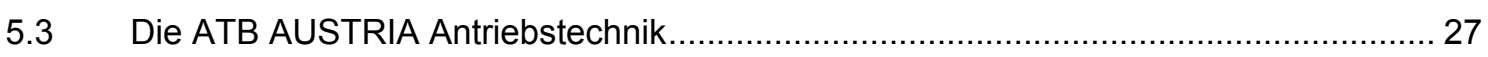

\section{Tabellenverzeichnis}

Tabelle $1 \quad$ Ausbildungslehrgänge des IKH in Osteuropa …................................................... 5

Tabelle 2 Themenbereiche des Lehrveranstaltungs- und Kursprogramms des IKH in Osteuropa.

Tabelle 3 Anzahl der Schulungsaktivitäten und Teilnahmen im Rahmen des

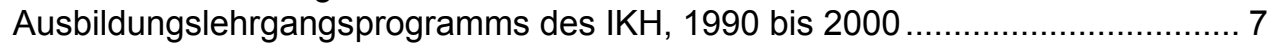

Tabelle $4 \quad$ Antwortquote der Unternehmensbefragung ................................................. 10 


\section{Grafikverzeichnis}

Grafik 1 Anzahl der Teilnahmen österreichischer und osteuropäischer Betriebe an Businessforen, 2000-2003, Werte geschätzt......

Grafik 2 Teilnahme der österreichischen Betriebe an Businessforen für unterschiedliche Länder, 2000-2003, Betriebe in Prozent.....

Grafik 3 Gründe für den vorzeitigen Abbruch bzw. das Nicht-Zustandekommen von Interviews, Betriebe in Prozent.

Grafik 4 Vergleich der Teilnahmefrequenz der Betriebe an Businessforen in der Grundgesamtheit und in der Stichprobe, nach Nationalität der eingeladenen ausländischen Unternehmen, Betriebe in Prozent

Grafik 5 Tätigkeitsbereich der befragten Unternehmen, Betriebe in Prozent 13

Grafik 6 Intensität der Geschäftsaktivitäten und Präsenz teilnehmender österreichischer Unternehmen im osteuropäischen Ausland, Betriebe in Prozent......

Grafik 7 Subjektiver Einschätzung des Kenntnisstandes der österreichischen Unternehmen hinsichtlich osteuropäischer Märkte, Betriebe in Prozent.

Grafik 8 Genutzte Informationsquellen der Betriebe für aktuelle osteuropäische Daten, Betriebe in Prozent

Grafik 9 Gründe für eine positive Teilnahmeentscheidung an den Businessforen, Betriebe in Prozent .

Grafik 10 Kontaktstufen bzw. Intensität der Kooperation im Zuge der Businessforen......

Grafik 11 Art der bei den Businessforen zustande gekommenen und anschließend verfestigten Kontakte, Betriebe in Prozent....

Grafik 12 Wichtigkeit der Kontakte für die Geschäftstätigkeit der österreichischen Unternehmen, Betriebe in Prozent

Grafik 13 Art und Anzahl abgeschlossener Verträge österreichischer Unternehmen mit osteuropäischen Partnern.

Grafik 14 Indirekte, durch Vertragsabschluss realisierte Auswirkungen, Betriebe absolut

Grafik 15 Nutzen der Businessforen für die österreichischen Betriebe, Durchschnittswerte

Grafik 16 Identifizierte Problembereiche bei der Zusammenarbeit mit osteuropäischen Unternehmen, Betriebe in Prozent

Grafik 17 Standorte der BRAMAC GmbH, Stand 11.12.2003. 


\section{Zusammenfassung und Schlussfolgerungen}

Bei der vorliegenden Studie handelt es sich um die Evaluierung der Auswirkungen der Businessforen des Wirtschaftsförderungsinstituts (WIFI) Österreich auf die österreichische Wirtschaft. Prozessorientierte und inhaltliche Aspekte werden im Gegensatz zu einer formativen Evaluierung nur am Rande behandelt. Die Arbeit stützt sich auf eine telefonische Vollerhebung bei 234 österreichischen Unternehmen, die in den Jahren 2000 bis 2003 an Businessforen teilgenommen haben, eine Dokumentenanalyse sowie persönliche Gesprächen mit Programmverantwortlichen. Die Antwortquote der telefonischen Erhebung lag bei ca. $26 \%$, d. h. dass Antworten von 61 Betrieben in die Analyse eingeflossen sind. Drei Unternehmen werden als „Good Practice“-Fallbeispiele dargestellt.

Die Businessforen sind Teil eines umfassenderen, seit dem Jahr 1990 laufenden Programms des WIFI, das das Zustandekommen von Geschäftskontakten zwischen osteuropäischen und österreichischen Unternehmen fördert. Dieses Programm besteht zum einen aus der Organisation von Lehrgängen in Osteuropa, mit denen Know-How Defizite bei der Anwendung moderner betriebswirtschaftlicher Methoden der ausländischen Unternehmen abgebaut und eine gewisse Affinität zu Österreich hinsichtlich möglicher Unternehmenskooperationen hergestellt werden sollen und zum anderen aus den besagten Businessforen, die in Österreich stattfinden und österreichischen Unternehmen die Gelegenheit geben, osteuropäische Manager/innen, die erfolgreich die WIFI Lehrgänge absolviert haben, kennen zu lernen und mit ihnen Geschäftsbeziehungen aufzubauen.

Die Anzahl der Teilnahmen österreichischer und osteuropäischer Unternehmen an den Businessforen konnte für den Untersuchungszeitraum nicht exakt ermittelt werden. Tendenziell war jedoch festzustellen, dass überproportional viele österreichische Betriebe Businessforen mit südosteuropäischen Unternehmen besucht haben. Dies spiegelt sich, mit Ausnahme einiger Länder, in verstärkter Form auch in der Stichprobe wider, was auf eine strategische Ausrichtung der teilnehmenden österreichischen Unternehmen auf diese Region hindeutet.

Die teilnehmenden österreichischen Unternehmen verfügen meist über überdurchschnittlich hohe Kenntnisse der für sie interessanten ausländischen Märkte und sind auch geschäftlich sehr aktiv im Ausland tätig (so verfügen beispielsweise rd. $47 \%$ der Betriebe über eigene ausländische Niederlassungen). Wichtigstes Teilnahmemotiv an den Businessforen ist für die österreichischen Unternehmen die Suche nach möglichen Kooperationspartner/innen, gefolgt von der Erweiterung der Marktkenntnisse. Die relativ hohe Bedeutung der Marktkenntnisse überrascht ob der hohen Einschätzung des eigenen Wissensstands und zeigt, dass trotz intensiver Recherchetätigkeit viele wichtige Information nicht oder nur schwer erhältlich sind. Für ihre Informationsrecherchen greifen die Betriebe am häufigsten auf die Services der Wirtschaftskammer Österreich, auf das Internet und auf Recherchen von eigenen Mitarbeiter/innen im Ausland zurück.

Insgesamt haben die 61 befragten Unternehmen 174 Mal ein Businessforum besucht. Von den 61 Betrieben konnten 8 keine Kontakte bei den Businessforen knüpfen. 53 hatten während der Veranstaltungen Kontakte mit ausländischen Unternehmen, 35 hiervon auch danach. 13 dieser 35 Betriebe konnten schließlich Verträge mit ausländischen Partnern abschließen. Jene 22 Unternehmen die mehrfach Kontakte hatten, aber (noch) keine Kontrakte abschließen konnten, sehen die Kontakte zu rd. $56 \%$ als „wichtig“ und zu ca. $28 \%$ als „eher wichtig“ für ihre Geschäftstätigkeit an. Etwa $60 \%$ planen, langfristig mit den ausländischen Partnern zusammenzuarbeiten. Kundenkontakte waren die häufigste Kontaktart dieser 22 Unternehmen. Damit wird offensichtlich, dass primär neue Absatzmöglichkeiten für eigene Produkte gesucht werden und weniger Produktionskooperationen oder Zulieferungen. 
Die 13 Unternehmen mit Vertragsabschlüssen haben insgesamt etwa 51 Kontrakte unterzeichnet. Die Mehrheit dieser Verträge waren Lieferverträge. 6 der 13 Unternehmen konnten das auf Grund der Businessforen zustande gekommene Vertragsvolumen schätzen. Gemäß ihren Angaben betrug das Vertragsvolumen insgesamt ca. $€ 17$ Mio. $€ 15$ Mio hiervon sind auf die Umsätze eines einzelnen Betriebs zurückzuführen. $€ 100.000$,- der $€ 17$ Mio wären ohne Teilnahme an den Businessforen nicht realisiert worden (reine Additionalitätseffekte), während $€ 250.000$,- an Vertragsvolumen nach Angaben der Unternehmen auch ohne die Businessforen zustande gekommen wären (reine Mitnahmeeffekte).

Der Großteil der Umsätze (€ 16,65 Mio) wäre ohne Besuch der Businessforen nur mit höherem Aufwand und/oder mit anderen Kooperationspartnern realisiert worden. Daraus ist ersichtlich, dass die Businessforen für die Geschäftsanbahnung vor allem die Rolle eines Katalysators spielen. Weiteres gaben 10 der 13 Unternehmen an, dass die vertraglich realisierten Kooperationen dazu beigetragen haben, die Wettbewerbsfähigkeit ihres Betriebs zu verbessern, 7 Betriebe meldeten Exportsteigerungen und 4 meinten, dass die Kooperation(en) Arbeitsplätze sichern halfen. 10 Unternehmen gehen davon aus, dass auch in Zukunft Zusatzumsätze mit den Partnerfirmen lukriert werden. Alle 13 Unternehmen planen auch in Zukunft mit den osteuropäischen Betrieben zu kooperieren.

Die Bewertung unterschiedlicher Aspekte in Hinblick auf den Nutzen der Teilnahme an den Businessforen durch die Unternehmen ergibt ein relativ homogenes Bild. Die Bewertungen liegen im Durchschnitt zwischen 2,6 (höhere Bekanntheit des eigenen Unternehmens) und 2,2 (bessere Kenntnisse bezüglich ausländischer Geschäftspraktiken) auf einer Skala von 1 = gar kein Nutzen bis $4=$ sehr hoher Nutzen. Der wesentlichste Problembereich bei Kooperationen mit osteuropäischen Unternehmen ist das fehlende Know-how (von 72 \% der österreichischen Betriebe als Problemfeld genannt), vor den Schwierigkeiten bei der Beschaffung konkreter Informationen (ca. $66 \%$ ). Sprachliche Verständigungsschwierigkeiten treten hingegen seltener auf (ca. $25 \%$ ). Fast $95 \%$ der befragten Unternehmen würden wieder an einem Businessforum teilnehmen.

Insgesamt zeigt sich, dass die Businessforen des WIFI das Potenzial haben, deutliche Impulse und Hilfestellungen für die Anbahnung von Geschäftkontakten zwischen österreichischen und osteuropäischen Unternehmen zu liefern. Die Tätigkeiten im Rahmen der Kursprogramme im Ausland werden prinzipiell von den österreichischen Betrieben, auf Grund der in Osteuropa vorhandenen Defizite, als sinnvoll und zieladäquat angesehen. Die festgestellten direkten Wirkungen (ca. $€ 17$ Mio an Vertragsvolumen, das in Folge der Businessforen zustande gekommen ist) wie auch die indirekten Wirkungen (Sicherung der Wettbewerbsfähigkeit, Wichtigkeit der Kontakte für die Geschäftstätigkeit) sind auf dem ersten Blick durchaus respektabel.

Bei genauerem Hinsehen ist jedoch festzuhalten, dass nur eine sehr kleine Zahl von Unternehmen für diese positiven Wirkungen verantwortlich ist. Ein wesentliches Ergebnis ist des Weiteren, dass hauptsächlich sehr auslandserfahrene Unternehmen am Programm teilnehmen und Betriebe, die in Osteuropa erst Fuß fassen wollen, kaum angesprochen werden.

Es gibt zahlreiche Indizien, dass durch eine verbesserte Programmorganisation, die insbesondere auf eine Intensivierung der Vorarbeiten zu den Businessforen abzielt, sowohl die Teilnahmehäufigkeit an den Businessforen (auch unter Einbeziehung „unerfahrener" Betriebe) als auch die Erfolgsquote (z.B. in Hinblick auf die Anzahl getätigter Abschlüsse) erhöht werden können. Verbesserungspotenzial zeigt sich vor allem auch in der Verwaltung der Datenbestände. 


\section{Einleitung}

Mit dem Beitritt von 10 Ländern - großteils aus Osteuropa - zur Europäischen Union am 1. Mai 2004 findet eine Entwicklung, die mit dem Fall des eisernen Vorhangs Ende der 80er Jahre begann, einen vorläufigen Höhepunkt. Das Ende der kommunistischen Ära, die Einführung der Marktwirtschaft und die Annäherung an den Westen Anfang der 90er Jahre eröffneten (und eröffnen noch immer) enorme Absatz- und Expansionsmöglichkeiten für österreichische Unternehmen auf den bislang schwer zugänglichen und (im marktwirtschaftlichen Sinne) wenig entwickelten Märkten Osteuropas. Die dynamische Entwicklung des Außenhandels Österreichs mit Ländern wie Ungarn, Rumänien, Bulgarien und Polen in den letzten Jahren zeigt, dass der osteuropäische Raum zu einem wesentlichen Wachstumsmotor für die österreichische Wirtschaft geworden ist.

Den Chancen, die sich österreichischen Betrieben in Osteuropa (nicht zuletzt auch auf Grund einer gemeinsamen Vergangenheit in der Monarchie) bieten stehen aber auch eine Reihe von Herausforderungen und Risiken gegenüber. Hierzu zählen u. a. mangelnde Kenntnisse über die ausländischen Märkte, Sprachprobleme, geringe Kenntnisse über mögliche Kooperations- und Ansprechpartner, aber auch fehlende Erfahrungen osteuropäischer Unternehmen im Umgang mit den neuen Marktformen, speziell bei der Anwendung moderner betriebswirtschaftlicher Methoden.

Bereits früh (seit Anfang der 90er Jahre) hat die Abteilung Internationaler Know-how Transfer (IKH) des Wirtschaftsförderungsinstituts (WIFI) der Wirtschaftskammer Österreich versucht, österreichischen Betrieben die Suche nach Kooperationspartnern in Osteuropa zu erleichtern. Mit Hilfe von Ausbildungslehrgängen in Osteuropa, die das IKH organisiert, sollen einerseits Know-how Defizite ausländischer Unternehmen in betriebswirtschaftlichen Belangen abgebaut und anderseits eine stärkere Anbindung an Österreich realisiert werden. Die Außenwirtschaftsorganisation (AWO) der Wirtschaftskammer Österreich organisiert schließlich Businessforen mit interessierten österreichischen und ausgewählten osteuropäischen Unternehmen, die an den Ausbildungslehrgängen teilgenommen haben. Bei den Businessforen handelt es sich um Veranstaltungen in Österreich, bei denen die Anbahnung von grenzüberschreitenden Geschäftskontakten im Vordergrund steht. Die Businessforen sind prinzipiell als integraler Teil des Ausbildungslehrgangsprogramms zu verstehen.

Vor diesem Hintergrund wurde die KMU FORSCHUNG AUSTRIA beauftragt, die Auswirkungen des Ausbildungslehrgangsprogramms bzw. konkreter der Businessforen auf die österreichische Wirtschaft zu erheben und zu evaluieren. Im Vordergrund stehen dabei sowohl die direkten Auswirkungen, wie beispielsweise die Anzahl getätigter Geschäftsabschlüsse, als auch die indirekten Auswirkungen, wie z.B. der Nutzen der Unternehmen im Hinblick auf die Erweiterung von Marktkenntnissen etc. Im Gegensatz zu einer formativen Evaluierung wurden prozess- und inhaltsorientierte Fragestellungen, z.B. für eine etwaige Optimierung der Programmorganisation, nur in so weit behandelt, als es für die Analyse der Auswirkungen zweckdienlich erschien.

Der vorliegende Bericht gliedert sich in folgende Abschnitte:

- In Abschnitt 2 werden die methodische Vorgehensweise erläutert und wichtige Kenngrößen der Stichprobenerhebung dargestellt.

- Abschnitt 3 beschreibt den Evaluationsgegenstand in einem Detaillierungsgrad, der für das Verständnis der Funktionsweise des Programms und für die Analyse möglicher Gründe hinsichtlich der Art und des Umfangs der festgestellten Auswirkungen notwendig ist.

- In Abschnitt 4 werden die Auswirkungen des Programms auf die österreichische Wirtschaft durchleuchtet.

- In Abschnitt 5 werden drei Unternehmen dargestellt, die von den Businessforen überdurchschnittlich profitiert haben und daher als "Good Practice" Beispiele angesehen werden können. 


\section{Beschreibung des Evaluierungsgegenstandes}

\subsection{Zielsetzungen und organisatorischer Aufbau des Programms}

Das Ausbildungslehrgangsprogramm des WIFI basiert auf zwei Säulen:

- der Durchführung von Lehrveranstaltungs- und Kursprogrammen für ausländische Unternehmen im Ausland und

- der Organisation von Businessforen mit (erfolgreich abschließenden) ausländischen Teilnehmer/innen der Lehrveranstaltungs- und Kursprogramme und interessierten österreichischen Betrieben im Inland.

Mit der Durchführung der Lehrgangs- und Kursprogramme in Osteuropa werden zwei Ziele verfolgt: Einerseits soll ausländischen Betrieben österreichisches Management Know-how vermittelt werden um Defizite bei der Anwendung moderner betriebswirtschaftlicher Methoden abzubauen. Andererseits soll über die Teilnahme an den Kursen eine Affinität zu Österreich aufgebaut werden, sodass österreichische Unternehmen in Partnerauswahlprozessen für Kooperationsvorhaben von den osteuropäischen Betrieben vorrangig angesprochen werden. Die Businessforen dienen der Zusammenführung osteuropäischer und österreichischer Firmen und damit der direkten Anbahnung von Geschäftskontakten. Außerdem soll die Bindung der osteuropäischen Manager/innen an Österreich (z.B. über Exkursionen etc.) nochmals verfestigt werden.

Die Kursprogramme und die Businessforen sind organisatorisch weitgehend getrennt. Für die Durchführung der Kursprogramme ist die Abteilung Internationaler Know-how Transfer (IKH) des WIFI zuständig. Diese arbeitet bei der Durchführung der Kurse mit Partnerinstituten vor Ort zusammen. Dabei finanziert das WIFI die Trainer/innenhonorare (auf Werksvertragsbasis) sowie die Reisekosten und stellt die Kursinhalte zusammen. Die ausländischen Partner übernehmen die Kosten für den Aufenthalt der Trainer/innen, übersetzen die Skripten und stellen die technische Infrastruktur sowie Dolmetscher/innen zur Verfügung. Außerdem ist das Partnerinstitut für die Vermarktung bzw. die Unternehmensansprache in Osteuropa zuständig, arbeitet aber hierbei mit der Außenwirtschaftsorganisation (AWO) der Wirtschaftskammer Österreich zusammen.

Die Businessforen werden (in der Regel) von der Außenwirtschaftsorganisation (AWO) der Wirtschaftskammer Österreich organisiert. Diese verfügt über Datenbanken mit österreichischen Unternehmen und ihren Geschäftsfeldern bzw. Interessensgebieten in Osteuropa. Die Ansprache der österreichischen Unternehmen erfolgt über schriftliche Aussendungen, die u a. auch eine Liste der osteuropäischen Teilnehmer/innen der Businessforen samt ihren Geschäftsfeldern und Kooperationsabsichten enthalten. Die osteuropäischen Teilnehmer/innen rekrutieren sich aus jenen Kursteilnehmer/innen, die einen Managementlehrgang positiv abgeschlossen haben. In Österreich sind neben der AWO auch Mitarbeiter/innen der IKH bei den Businessforen anwesend.

\subsection{Inhalte und Abläufe des Programms}

Der Umfang der Lehrgangs- und Kursprogramme im Ausland variiert sehr stark und reicht vom 2 bis 3-tägigen Symposium über mehrwöchige Lehrgänge bis zur 18-monatigen Ausbildung zur Unternehmensberater/in (siehe Tabelle 1). Zu Beginn der Programmlaufzeit Anfang der 90-er wurden hauptsächlich kürzere Spezialveranstaltungen und Symposien durchgeführt während nunmehr schwerpunktmäßig längerfristige Lehrgänge und Schulungen organisiert werden. 
Tabelle $1 \quad$ Ausbildungslehrgänge des IKH in Osteuropa

\begin{tabular}{|c|c|c|}
\hline Art der Veranstaltung & Dauer & Inhalt \\
\hline Symposium & 2-3 Tage & $\begin{array}{l}\text { Einführungsveranstaltungen zu diversen } \\
\text { Themengebieten }\end{array}$ \\
\hline Spezialveranstaltungen & 3-7 Tage & Basisausbildung mit weiterer Vertiefung \\
\hline Managementlehrgänge & 18-30 Tage & $\begin{array}{l}\text { Lehrgänge mit Prüfungsabschluss; erfolgreich } \\
\text { abschließende Unternehmen werden zur Vertiefung } \\
\text { nach Österreich zu Business Foren eingeladen }\end{array}$ \\
\hline Trainerschulung & $6-10$ Tage & Schulung von Vortragenden \\
\hline $\begin{array}{l}\text { Ausbildung zur } \\
\text { Unternehmensberate/inr }\end{array}$ & 18 Monate & $\begin{array}{l}\text { Unternehmensberater/innenausbildung nach } \\
\text { österreichischem Vorbild mit entsprechendem } \\
\text { Abschluss }\end{array}$ \\
\hline
\end{tabular}

Grundsätzlich wird versucht, den Betrieben „Hilfe zur Selbsthilfe“ zu vermitteln. Im Speziellen sollen privates Unternehmertum und leistungsorientiertes Selfmanagement bei den osteuropäischen Manager/innen als wichtige Prinzipien der Unternehmenskultur verankert werden, reines Faktenwissen steht nicht im Vordergrund. Die Kurse und Lehrgänge decken hierfür eine weite Bandbreite an Themen ab (siehe Tabelle 2).

Tabelle 2 Themenbereiche des Lehrveranstaltungs- und Kursprogramms des IKH in Osteuropa

Kurse und Lehrgänge im Rahmen des Ausbildungslehrgangsprogramms des IKH

\begin{tabular}{l|l}
\hline $\begin{array}{l}\text { Managementlehrgang für Unternehmer und } \\
\text { Führungskräfte }\end{array}$ & Führung und Motivation von Mitarbeiter/innen \\
Managementkurs für Klein- und Mittelbetriebe & Personalwirtschaft \\
Betriebsgründung & Ausbildung zur Betriebsberater/in \\
Außenhandel für Klein- und Mittelebetriebe & Lehrgang zur Management-Ausbildner/in \\
Aufbau und Struktur von Joint-Venture Betrieben & Train-the-Trainer \\
Rechnungswesen und Finanzierung & Hotelmanagement \\
Finanzmanagement & Zeitmanagement \\
Controlling & Unternehmensbewertung \\
Strategische Unternehmensführung & Staatliche Wirtschaftsverwaltung unter \\
& marktwirtschaftlichen Bedingungen \\
Grundlagen des Marketing & Europäische Normung \\
Strategisches Marketing & Energiemanagement, Luftreinhaltung, \\
& Abfallwirtschaft \\
\hline
\end{tabular}

Quelle: Österreichisches Wirtschaftsförderungsinstitut 2001

Direkt nach Abschluss der Lehrgänge werden sowohl die Lehrgänge selbst als auch die Qualität der Vortragenden evaluiert. Hierzu hat das WIFI entsprechende Fragebögen erstellt, die von den Partnerinstituten in die jeweilige Landessprache übersetzt werden. Manager/innen, die die die Abschlussprüfung eines Managementlehrgangs positiv absolviert haben, werden nach Österreich zu einem Businessforum eingeladen. Zuvor müssen sie einen Fragebogen über ihr Unternehmen ausfüllen, der Angaben über Geschäftsfelder und Kooperationsabsichten enthält. 
Businessforen mit Unternehmen eines osteuropäischen Landes (meist auch aus ein oder zwei bestimmten Regionen bzw. Städten) werden ein bis zwei Mal jährlich organisiert. Die Veranstaltungen dauern, je nach Anzahl der Teilnehmer/innen, ein oder zwei Tage. Die Anreise wird von den Teilnehmer/innen selbst finanziert, das WIFI übernimmt die Kosten des Aufenthalts (Hotel auf Basis Nächtigung/Frühstück) und die Organisation des Rahmenprogramms.

Der Ablauf der Foren ist in weiten Zügen standardisiert. Einer Begrüßung durch den Vorsitzenden der AWO, in der auch kurz die Wirtschaftsbeziehungen Österreichs mit dem jeweiligen Land, z.B. an Hand der Entwicklung des Außenhandels, dargestellt werden, folgt die Aufteilung der Gruppe der ausländischen Teilnehmerinnen nach Regionen bzw. Städten in verschiedene angrenzende Räume. Jedem Unternehmen wird ein eigener Schreibtisch zur Verfügung gestellt, an dem die Manager/innen Platz nehmen. Beim Eingang der Räume liegt u.a. eine Liste der angemeldeten österreichischen Teilnehmer/innen mit den Gesprächsterminen für die osteuropäischen Betriebe auf.

Dolmetscher/innen sind anwesend und können jederzeit hinzugezogen werden. Meist ist auch noch eine offizielle Vertretung des Landes (z.B. die Botschaft oder eine Außenhandelsorganisation) zugegen, die bei bestimmten Fragestellungen Hilfestellungen anbietet. Die Gespräche sind für maximal einen Halbtag anberaumt. Nach Abschluss der Gespräche erfolgt die Abwicklung des Rahmenprogramms mit Exkursionen.

Sowohl österreichische als auch ausländische Teilnehmer/innen werden direkt nach dem Forum gebeten, einen Evaluationsfragebogen auszufüllen. Eine Befragung einige Zeit nach Besuch des Businessforums über Verhandlungsfortschritte etc. wird nicht durchgeführt.

\subsection{Der bisherige Programmverlauf in Zahlen}

Die Ausbildungslehrgänge werden vom WIFI seit dem Jahr 1990 organisiert. In der Zeit zwischen 1990 und 2000 wurden Lehrgänge in 29 Ländern durchgeführt, an denen rd. 60.000 ausländische Betriebe teilnahmen. Neben Osteuropa wurde das Programm auch in Ländern wie Südafrika oder China angeboten (siehe Tabelle 3).

Das Ausbildungslehrgangsprogramm wird von der österreichischen Bundesregierung und von der Wirtschaftskammer Österreich finanziert. Das Finanzierungsvolumen betrug für den Zeitraum 1990 bis 2000 rd. $€ 10,9$ Mio, dies entspricht einem jährlichen Budget von durchschnittlich ca. $€ 1$ Mio.

Insgesamt erscheint es mit vertretbarem Aufwand nicht möglich, die genaue Anzahl der teilnehmenden Betriebe bzw. der Teilnahmen an den Businessforen im Untersuchungszeitraum zu bestimmen. Als Grundlage kämen hierfür prinzipiell einerseits Teilnahmestatistiken und andererseits die Adressdatenbank der österreichischen Teilnehmer/innen in Frage. Beide Datenquellen weisen jedoch Unstimmigkeiten auf und lassen sich nicht in zufrieden stellender Weise für die Bestimmung der Teilnahmen abgleichen. 
Tabelle 3 Anzahl der Schulungsaktivitäten und Teilnahmen im Rahmen des Ausbildungslehrgangsprogramms des IKH, 1990 bis 2000

\begin{tabular}{|c|c|c|}
\hline Land & Veranstaltungen/Kurse & Anzahl der Teilnehmer/innen \\
\hline Albanien & 33 & 706 \\
\hline Aserbaidschan & 1 & 11 \\
\hline Bosnien-Herzegowina & 72 & 1.090 \\
\hline Bulgarien & 520 & 10.071 \\
\hline China & 13 & 270 \\
\hline CSFR & 104 & 2.862 \\
\hline DDR (1990) & 8 & 136 \\
\hline Estland & 26 & 239 \\
\hline Georgien & 16 & 238 \\
\hline Serbien-Montenegro & 16 & 342 \\
\hline Kasachstan & 18 & 231 \\
\hline Kroatien & 50 & 787 \\
\hline Lettland & 137 & 2.822 \\
\hline Litauen & 82 & 1.033 \\
\hline Mazedonien & 3 & 71 \\
\hline Moldawien & 62 & 926 \\
\hline Mongolei & 28 & 763 \\
\hline Palästina & 15 & 199 \\
\hline Polen & 432 & 9.750 \\
\hline Rumänien & 723 & 12.616 \\
\hline Russ. Förderation & 315 & 5.200 \\
\hline Slowakei & 42 & 776 \\
\hline Slowenien & 104 & 1.642 \\
\hline Südafrika & 10 & 315 \\
\hline Syrien & 4 & 62 \\
\hline Tschechische Republik & 102 & 1.497 \\
\hline Tunesien & 4 & 36 \\
\hline UdSSR & 27 & 603 \\
\hline Ukraine & 213 & 3.212 \\
\hline Ungarn & 119 & 1.516 \\
\hline Usbekistan & 15 & 430 \\
\hline SUMME & 3.314 & 60.452 \\
\hline
\end{tabular}

In der Teilnahmestatistik liegen Daten für den Zeitraum Jänner 2000 bis 13. Mai 2003 vor, die sich aus Auswertungen der Evaluationsfragebögen ergeben. Gemäß den Angaben aus diesen Unterlagen sollten 1.914 osteuropäische und 1.069 österreichische Betriebe an Businessforen teilgenommen haben. Insgesamt wären dabei 2.694 Geschäftskontakte zustande gekommen. Die Adressdatenbank des WIFI enthält hingegen die Kontaktdetails von lediglich 234 österreichischen Betrieben, die jeweils ein Businessforum besucht haben sollen. 
Grafik 1 Anzahl der Teilnahmen österreichischer und osteuropäischer Betriebe an Businessforen, 2000-2003*), Werte geschätzt

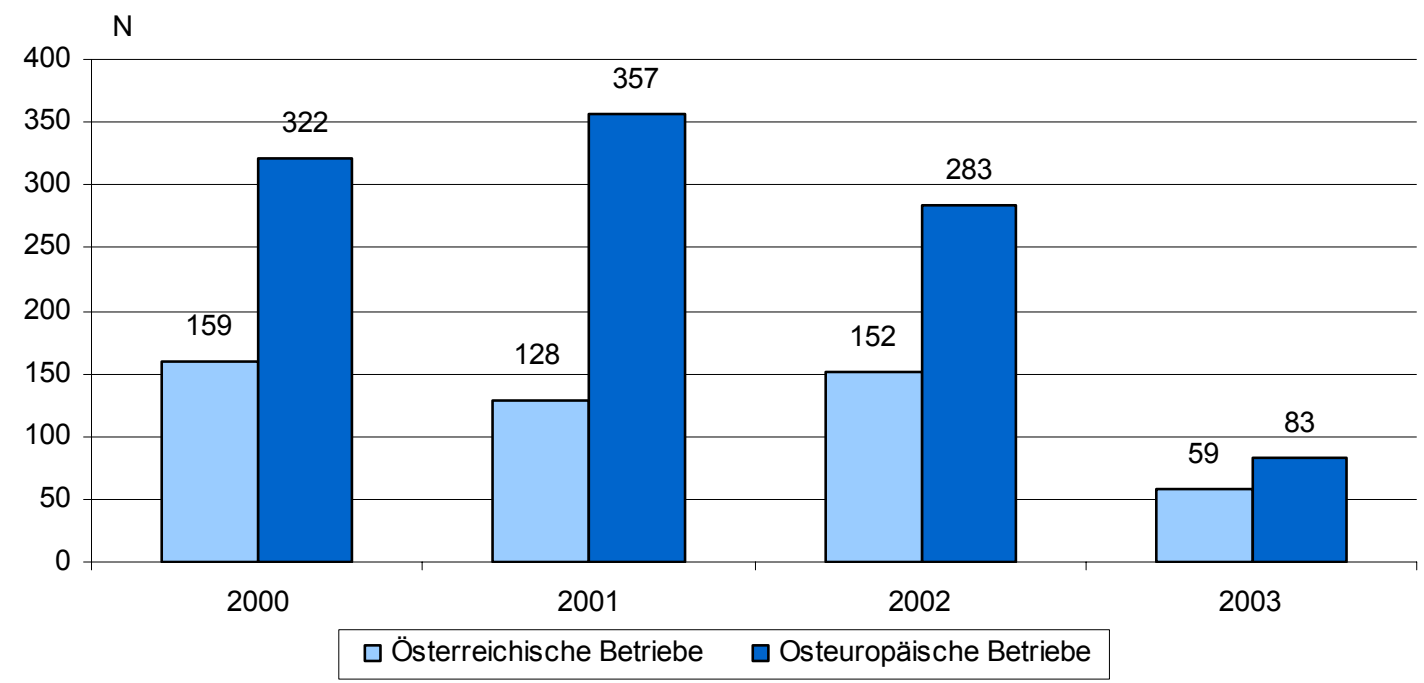

*) 2003: bis 13.5.2003

Quelle: KMU FORSCHUNG AUSTRIA; WIFI (2003): Teilnahmestatistik der Businessforen

Der Versuch des Datenabgleichs deutet darauf hin, dass in den Teilnahmestatistiken Betriebe, die an zweitägigen Veranstaltungen teilgenommen haben, doppelt gezählt wurden. Damit müssen die Werte der Teilnahmestatistiken deutlich nach unten revidiert werden. Des Weiteren haben offenbar viele österreichische Unternehmen an mehreren Businessforen teilgenommen, was aber in der Adressdatenbank nicht erfasst wurde. Die Anzahl der tatsächlich Teilnahmen läge damit (schätzungsweise um den Faktor $3^{1}$ ) über der Anzahl der Betriebe in der Adressdatenbank. Weiters muss berücksichtigt werden, dass einige Betriebe, die zwar eingeladen wurden, in der Folge aber nicht an den Businessforen teilgenommen haben trotzdem in der Adressdatenbank angeführt sind (siehe hierzu auch Kapitel 3). Schließlich fehlen in der Datenbank auch einige Adressen, z.B. von jenen Unternehmen die 2002 an einem russischen Businessforum teilgenommen haben.

Um dennoch zumindest indikative Aussagen treffen zu können, wurde die Teilnahmestatistik dahingehend korrigiert, dass bei zweitägigen Veranstaltungen lediglich das Minimum der angegebenen Anzahl der Teilnehmer/innen beider Tage berücksichtigt wurde. Die Wahl des Minimums an Stelle eines Mittelwertes ermöglicht eine bessere Vergleichbarkeit mit den Daten aus der Adressdatenbank.

Dadurch ergibt sich folgendes Bild (siehe Grafik 1): Die Anzahl der Teilnahmen österreichischer Betriebe wäre mit schätzungsweise 500 für den Zeitraum Januar 2000 bis Mai 2003 nur etwa halb so hoch wie die Teilnahmefrequenz der osteuropäischen Betriebe. Zudem zeigt sich, dass die Teilnahmefrequenz der ausländischen Betriebe in den Jahren 2002 und (hochgerechnet) 2003 leicht rückläufig tendiert.

Dieser Faktor ergibt sich aus der durchschnittlichen Teilnahmehäufigkeit der Betriebe, die bei der vorliegenden Evaluation mitgewirkt haben (siehe Abschnitt 4.2). Da anzunehmen ist, dass die Teilnahmehäufigkeit der nicht antwortenden Betriebe unter jener der antwortenden liegt, stellt die ermittelte Teilnahmehäufigkeit eine Obergrenze für eine evtl. Hochrechnung aus dem gesamten Adressmaterial dar. 
Grafik 2 Teilnahme der österreichischen Betriebe an Businessforen für unterschiedliche Länder, 2000-2003*), Betriebe in Prozent

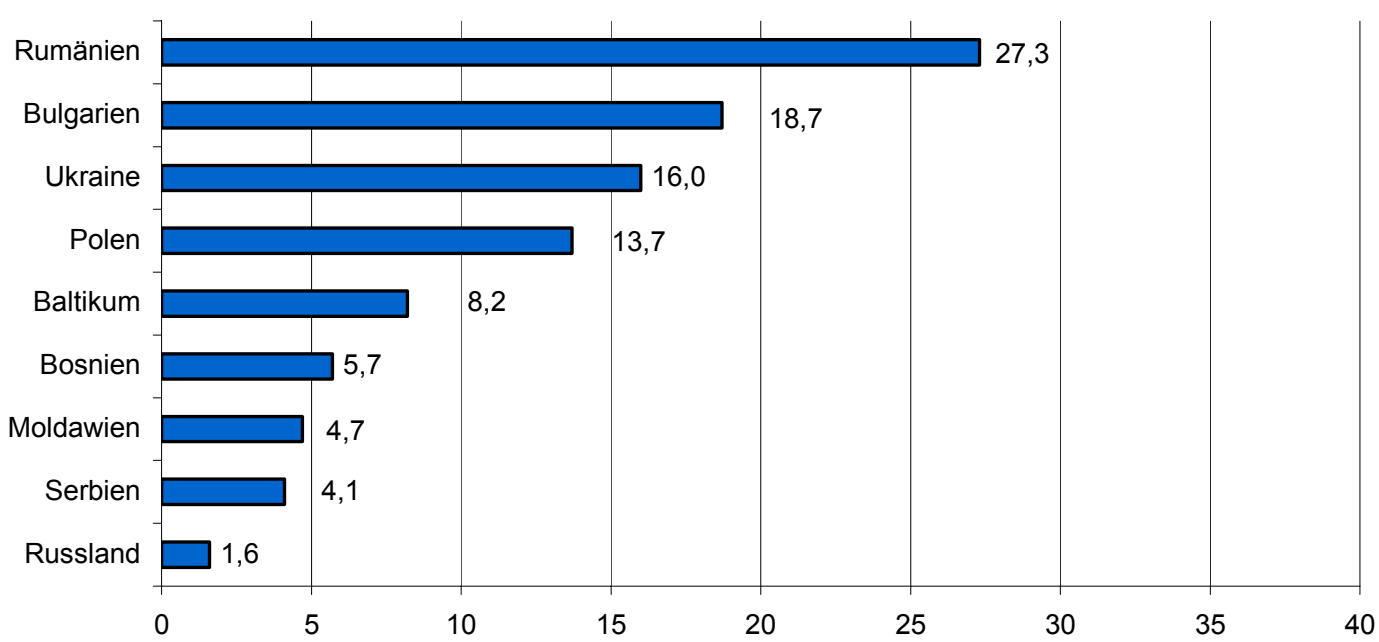

*) 2003: bis 13.5.2003

Quelle: KMU FORSCHUNG AUSTRIA; WIFI (2003): Teilnahmestatistik der Businessforen

Schwerpunktmäßig haben die österreichischen Unternehmen vor allem Businessforen mit Betrieben aus südosteuropäischen Ländern bzw. Ländern des Balkans besucht (siehe Grafik 2). An der Spitze steht hier Rumänien, auf dessen zugehörige Businessforen schätzungsweise mehr als ein Viertel aller Teilnahmen entfällt. 


\section{Methodische Vorgehensweise und Kenngrößen der empirischen Erhebung}

Ziel dieser Evaluierung ist die Erhebung der direkten und indirekten Auswirkungen der Businessforen in Österreich auf die österreichische Wirtschaft. Zur Messung dieser Auswirkungen wurde auf eine telefonische Befragung aller erfassten österreichischen Teilnehmer/innen der Businessforen aus den Jahren 2000 bis 2003 zurückgegriffen. Ergänzt wurde die Befragung durch vereinzelte persönliche Gespräche mit Stakeholdern und Programmmitarbeiter/innen, einer Dokumentenanalyse sowie der Identifikation und Darstellung von drei "Good Practice“Fallbeispielen.

Es sei jedoch darauf hingewiesen, dass die ausschließliche Konzentration auf die österreichischen Teilnehmer/innen der Businessforen den Nachteil mit sich bringt, dass positive Auswirkungen auf die österreichische Wirtschaft, die sich aus Lerneffekten der ausländischen Teilnehmer/innen ergeben (z. B. auf Grund eines höheren Kenntnisstandes über die österreichische Wirtschaft und damit verbunden einer höheren Bereitschaft, mit österreichischen Unternehmen zu kooperieren) nicht erhoben werden können.

Für die Unternehmensbefragung wurde eine telefonische Vollerhebung unter Zuhilfenahme eines standardisierten Interviewleitfadens bei den österreichischen Teilnehmer/innen der Business Foren durchgeführt. Die Größe der Grundgesamtheit, die sich aus der Anzahl der Eintragungen in der Adressdatenbank des WIFI ergab, lag bei 234 Betrieben. So fern es nicht möglich war, mit den Teilnehmer/innen bei der ersten telefonischen Kontaktaufnahme den Fragebogen durchzuarbeiten, wurde entweder ein späterer Termin vereinbart oder der Fragebogen per Fax bzw. E-Mail zum Ausfüllen versandt. Kontaktpersonen, die beim ersten Versuch der telefonischen Kontaktanbahnung nicht erreicht werden konnten, wurden zu einem späteren Zeitpunkt nochmals angerufen. Der Erhebungszeitraum erstreckte sich vom 1. Oktober 2003 bis zum 28. Oktober 2003.

Tabelle $4 \quad$ Antwortquote der Unternehmensbefragung

\begin{tabular}{l|r}
\hline Fragebögen & $\mathbf{n}$ \\
\hline Grundgesamtheit & 234 \\
Verwertbare Interviews & 61 \\
\hline \hline Antwortquote & $26,1 \%$ \\
\hline Quelle: KMU FORSCHUNG AUSTRIA & \\
\hline
\end{tabular}

Insgesamt wurden 61 Interviews erfolgreich durchgeführt. Bezogen auf die Grundgesamtheit von 234 Unternehmen entspricht dies einer Rücklauf- oder Antwortquote von 26,1 \%. 173 Interviews sind entweder nicht zu Stande gekommen oder mussten vorzeitig abgebrochen werden. 
Grafik 3 Gründe für den vorzeitigen Abbruch bzw. das Nicht-Zustandekommen von Interviews, Betriebe in Prozent

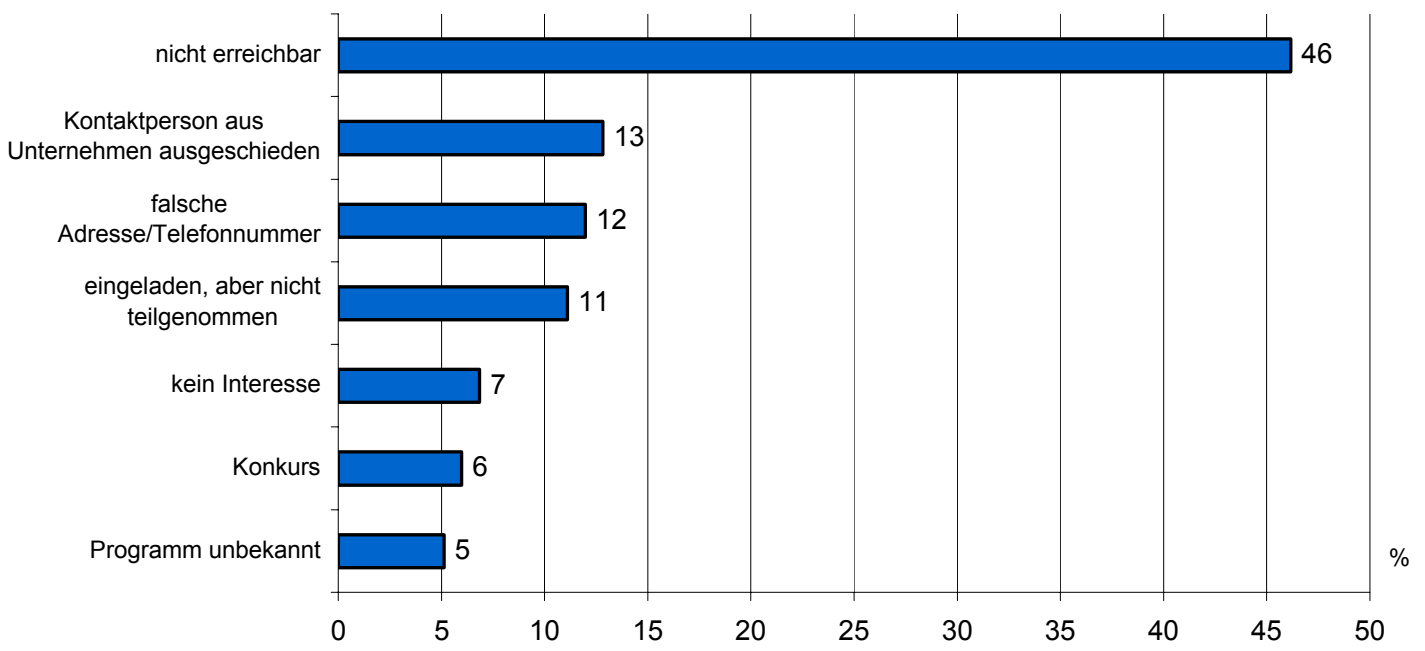

Quelle: KMU FORSCHUNG AUSTRIA

Die Gründe für den vorzeitigen Abbruch bzw. das Nicht-Zustandekommen der Interviews stellen sich wie folgt dar: Rd. $46 \%$ der Kontaktpersonen konnten selbst nach teilweise mehrmaligen Versuchen nicht erreicht werden. In rd. $13 \%$ der Fälle ist die entsprechende Kontaktperson aus dem Unternehmen ausgeschieden. Bei $12 \%$ der erfolglos kontaktierten Betriebe waren die Einträge in der Adressdatenbank fehlerhaft. Zudem stellte sich heraus, dass eine Reihe von Unternehmen (ca. $11 \%$ der nicht antwortenden Betriebe) in die Adressdatenbank aufgenommen wurden, die zwar zu einem Businessforum eingeladen waren, letztlich aber an diesem nicht teilgenommen haben.

Rd. $7 \%$ hatten kein Interesse, an der Befragung teilzunehmen, weitere $6 \%$ der Betriebe in der Adressdatenbank des WIFI existieren nicht mehr. Ca. $5 \%$ der nicht antwortenden Unternehmen war schließlich das WIFI Programm gänzlich unbekannt bzw. konnten sich die angegebenen Kontaktpersonen nicht mehr an eine Teilnahme erinnern.

Insgesamt haben die befragten Unternehmen ca. 174 Mal an den Businessforen teilgenommen, d.h. ein Unternehmen hat im Durchschnitt fast drei Veranstaltungen besucht. Frequentiert wurden schwerpunktmäßig Veranstaltungen mit Betrieben aus den Balkanstaaten bzw. aus Südosteuropa (siehe Grafik 4). Im Vergleich zur Teilnahmestruktur in der Grundgesamtheit zeigt sich, dass vereinzelte Länder in der Stichprobe deutlich unter- bzw. überrepräsentiert sind. 
Grafik $4 \quad$ Vergleich der Teilnahmefrequenz der österreichischen Betriebe an Businessforen in der Grundgesamtheit und in der Stichprobe, nach Nationalität der eingeladenen ausländischen Unternehmen, Betriebe in Prozent

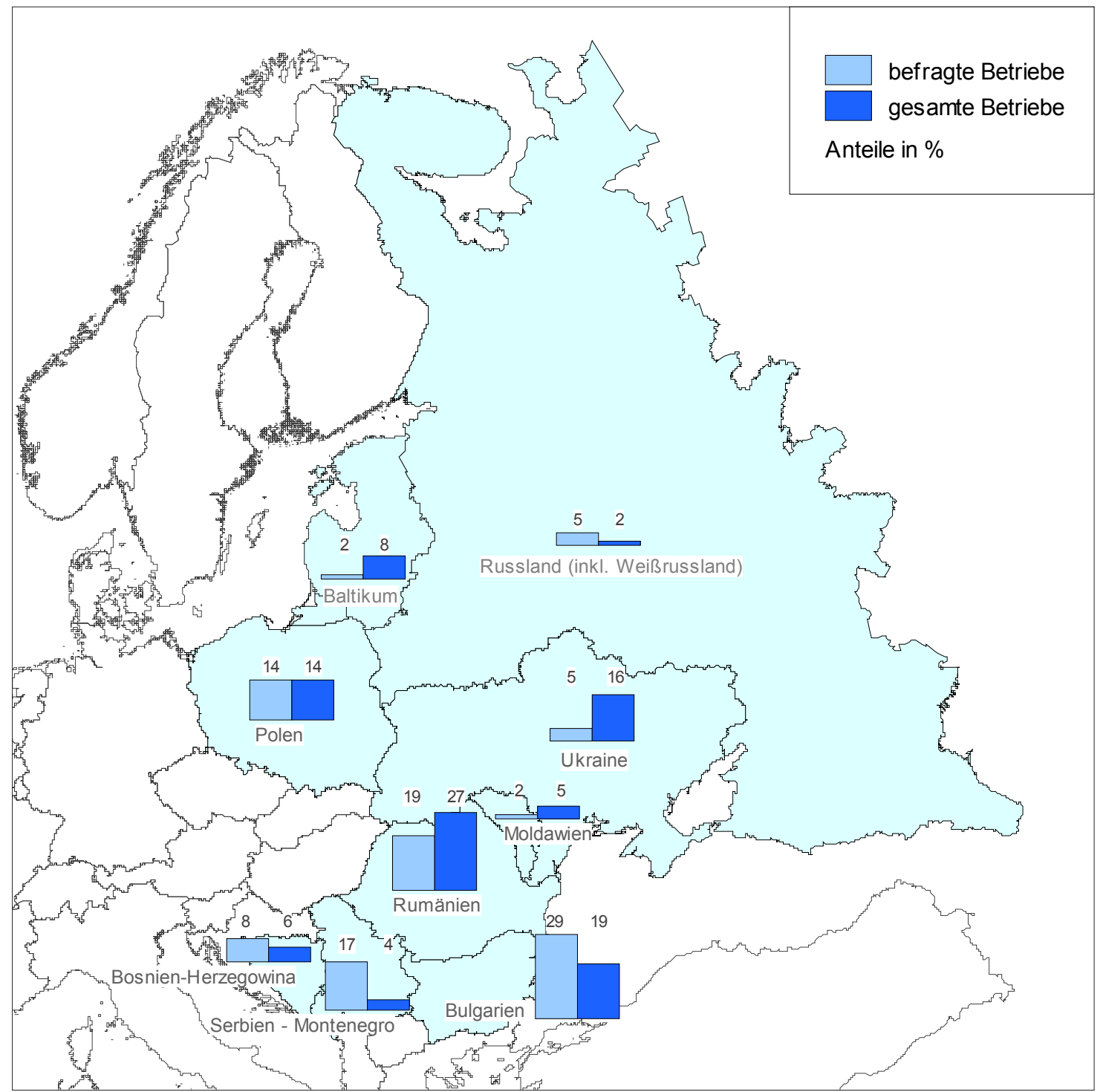

Quellen: KMU FORSCHUNG AUSTRIA, Unternehmensbefragung; WIFI (2003): Teilnahmestatistik der Businessforen

Am häufigsten wurden Businessforen mit bulgarischen und rumänischen Betrieben besucht. Während Teilnehmer/innen bulgarischer Businessforen jedoch prozentuell häufiger in der Stichprobe als in der Grundgesamtheit anzutreffen sind, verhält es sich bei rumänischen Businessforen genau umgekehrt. Besonders offensichtlich sind die Differenzen bei serbischen Businessforen. Nach der offiziellen Teilnahmestatistik zählen diese Veranstaltungen zu den weniger häufig besuchten, in der Stichprobe liegen sie allerdings an dritter Stelle. Umgekehrt verhält es sich mit ukrainischen Businessforen: Hier stehen relativ hohen Teilnahmezahlen in der Grundgesamtheit verhältnismäßig wenige Teilnahmen in der Stichprobe gegenüber. Ein ähnliches Bild bieten auch die Teilnehmerzahlen der Businessforen aus den baltischen Ländern, deren Anteil in der Stichprobe sehr gering ist. 
Eine Analyse der teilnehmenden Unternehmen zeigt, dass besonders jene, die ein rumänisches, bulgarisches, bosnisches oder serbisches Businessforum besucht haben, häufig auch an Businessforen der anderen Balkan- bzw. südosteuropäischen Länder teilgenommen haben. Daraus lässt sich allgemein eine strategische Ausrichtung dieser Unternehmen in Richtung Balkan/Südosteuropa ableiten.

\section{Grafik 5 Tätigkeitsbereich der befragten Unternehmen, Betriebe in Prozent}

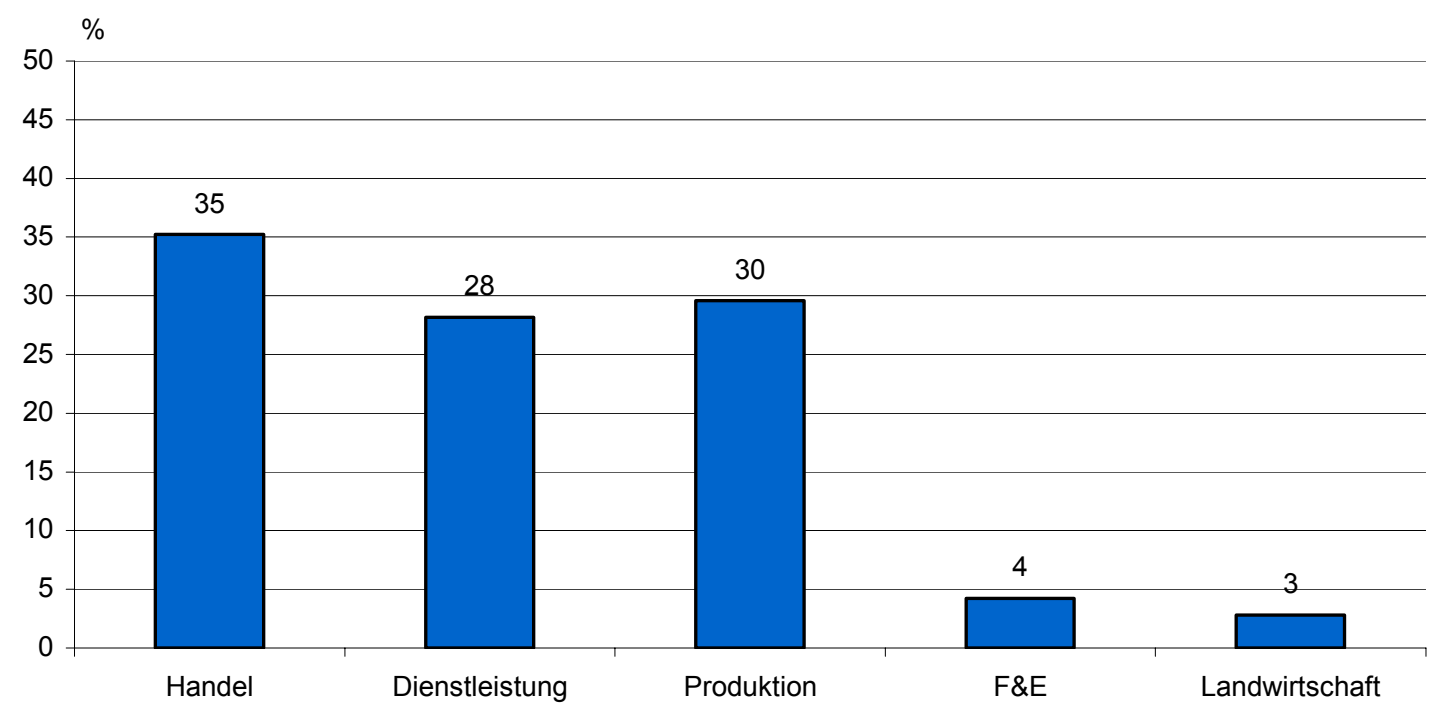

Quelle: KMU FORSCHUNG AUSTRIA, Unternehmensbefragung

Der Tätigkeitsbereich der an den Businessforen teilnehmenden Betriebe ist sehr heterogen (siehe Grafik 5). Rd. $35 \%$ sind im Handel tätig, bei ca. $30 \%$ handelt es sich um produzierende Betriebe der Industrie oder des Gewerbes und rd. $28 \%$ sind Dienstleistungsunternehmen. Gering ist jedenfalls der Anteil der Betriebe, deren Tätigkeitsschwerpunkt in der Forschung und Entwicklung (rd. 4 \%) und in der Landwirtschaft (rd. $3 \%$ ) liegt. 


\section{Analyse der Auswirkungen der Business Foren}

\subsection{Bisherige Auslandserfahrungen der teilnehmenden Unternehmen}

Die an den Businessforen teilnehmenden österreichischen Unternehmen sind in Osteuropa großteils geschäftlich sehr aktiv tätig. Die hohe Intensität der Auslandsaktivitäten zeigt sich vor allem daran, dass nahezu die Hälfte der Betriebe (rd. 48 \%) über eine eigene Niederlassung im osteuropäischen Ausland verfügt (siehe Grafik 6). Etwa ein Viertel der Betriebe kann auf ausländische Vertriebspartner zurückgreifen. Ca. ein Fünftel kooperiert mit zumindest einem ausländischen Unternehmen. Vereinzelte Geschäftskontakte bestehen des Weiteren bei rd. $21 \%$ der österreichischen Betriebe. $5 \%$ halten Beteiligungen an ausländischen Betrieben. Lediglich ein geringer Anteil (rd. $2 \%$ der befragten Unternehmen) ist bislang noch nicht im osteuropäischen Ausland geschäftlich aktiv geworden.

Grafik 6 Intensität der Geschäftsaktivitäten und Präsenz teilnehmender österreichischer Unternehmen im osteuropäischen Ausland, Betriebe in Prozent *)

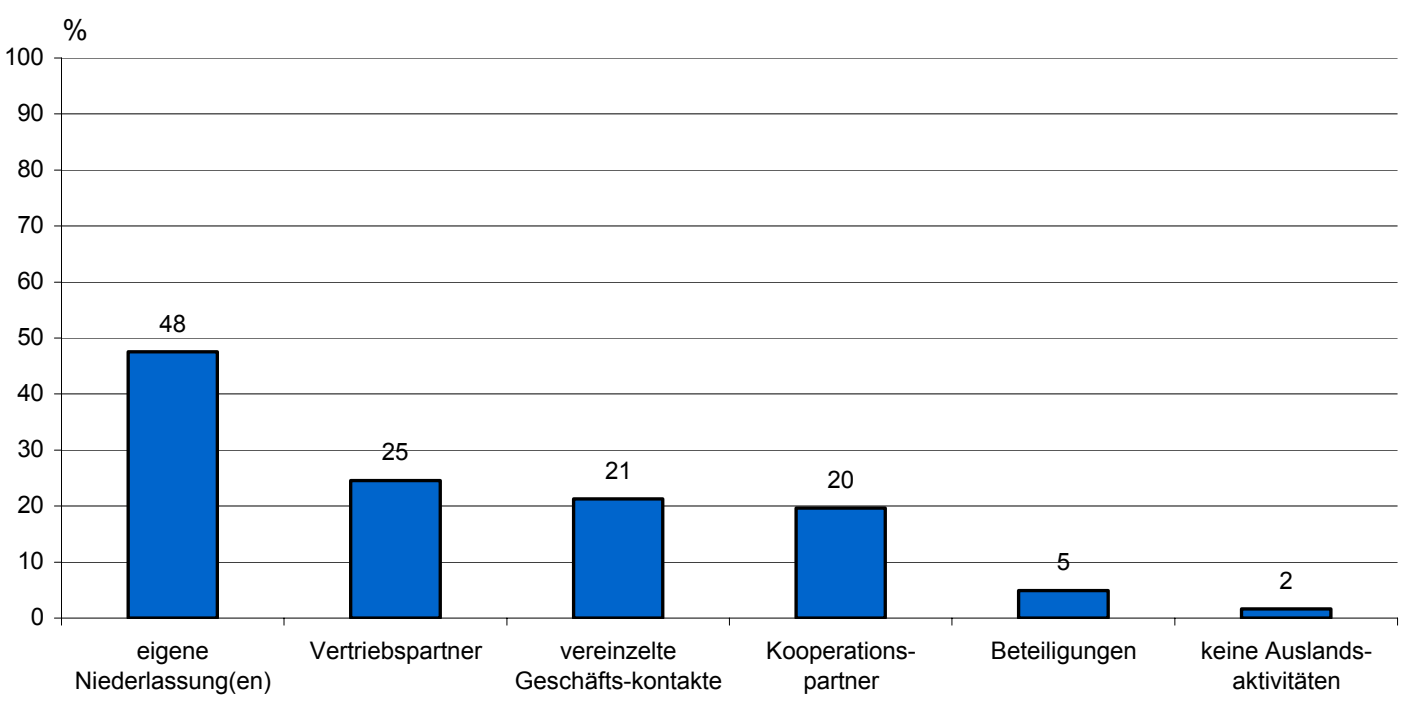

Quelle: KMU FORSCHUNG AUSTRIA, Unternehmensbefragung

*) Mehrfachnennungen möglich

Bedingt durch das intensive Auslandsengagement ist auch der Kenntnisstand der österreichischen Unternehmen über osteuropäische Märkte sehr hoch. Ca. 43 \% der österreichischen Betriebe geben an, über für sie interessante osteuropäische Märkte „sehr gut“ Bescheid zu wissen, weitere $46 \%$ stufen ihren Kenntnisstand als "gut" ein. Rd. $10 \%$ bewerten ihren Wissensstand zumindest als „ausreichend“ und nur ein kleiner Anteil von etwa $2 \%$ glaubt, „ungenügend“ über osteuropäische Märkte informiert zu sein. 
Grafik 7 Subjektiver Einschätzung des Kenntnisstandes der österreichischen Unternehmen hinsichtlich osteuropäischer Märkte, Betriebe in Prozent

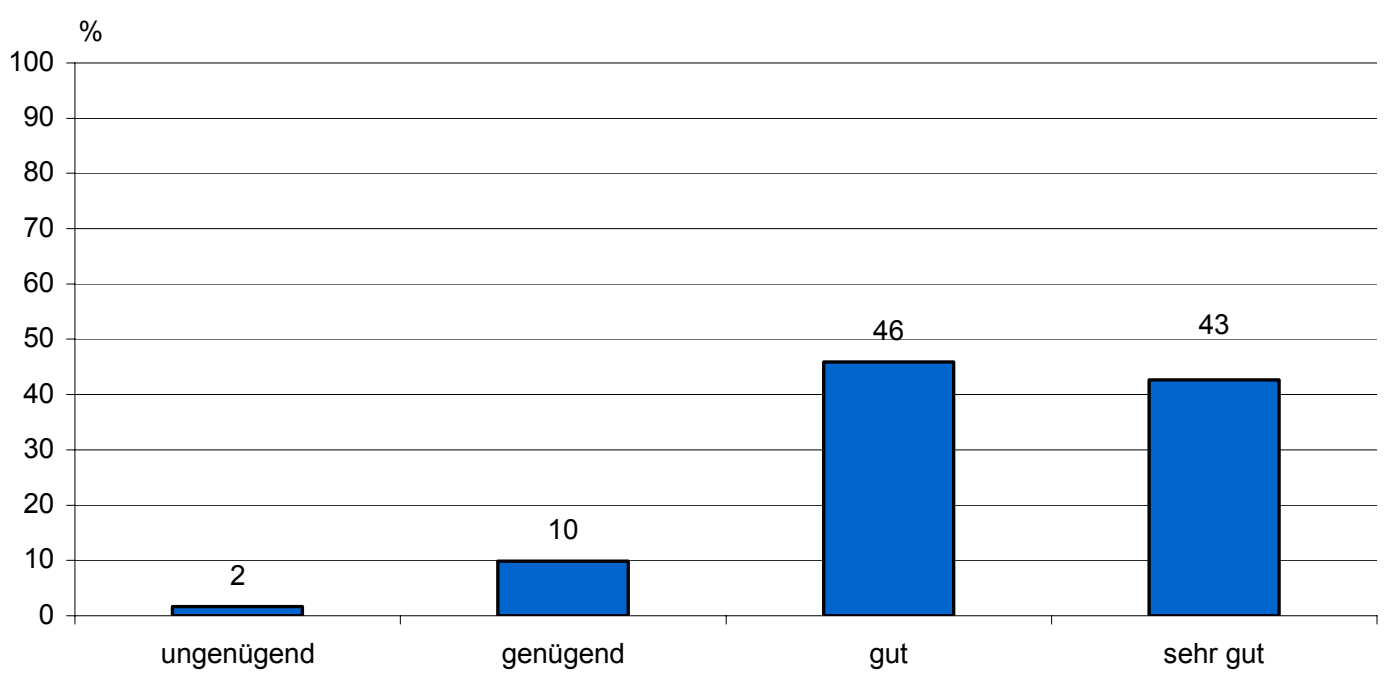

Quelle: KMU FORSCHUNG AUSTRIA, Unternehmensbefragung

Zur Beurteilung aktueller Entwicklungen und zur Analyse vorhandener Strukturen auf osteuropäischen Märkten greifen die Manager/innen der österreichischen Programmteilnehmer auf eine Vielzahl von Informationsquellen zurück (siehe Grafik 8). Am beliebtesten sind hierbei die Informationsservices der Wirtschaftskammer Österreich, die ca. 9 von 10 Unternehmen nutzen. Rd. $80 \%$ verwenden das Internet als Informationsquelle.

Grafik 8 Genutzte Informationsquellen der Betriebe für aktuelle osteuropäische Daten, Betriebe in Prozent *)

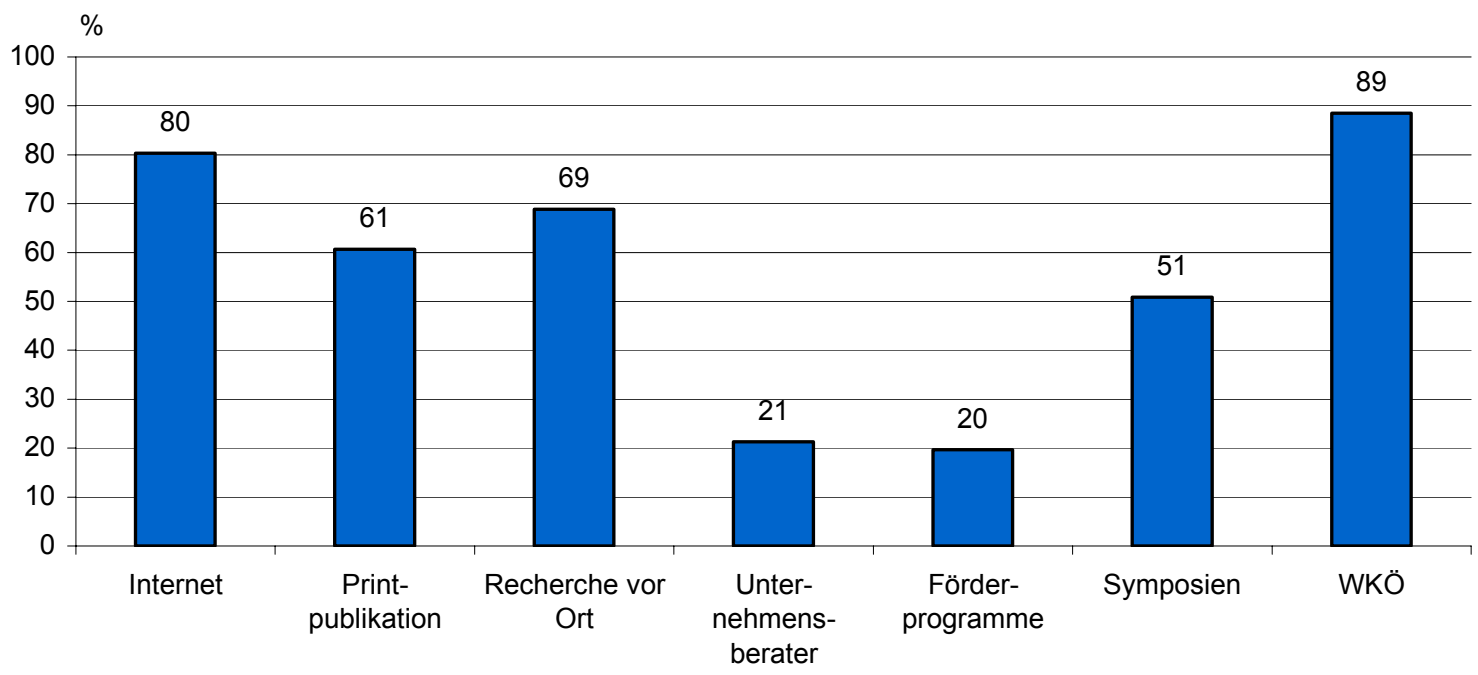

*) Mehrfachnennungen möglich

Quelle: KMU FORSCHUNG AUSTRIA, Unternehmensbefragung 
Sehr hoch (ca. $69 \%$ ) ist auch der Anteil jener Betriebe, die Recherchen von Mitarbeiter/innen vor Ort durchführen lassen - eine direkte Folge der starken Auslandspräsenz mit eigenen Niederlassungen und Vertriebspartnerschaften. Rd. $61 \%$ der Teilnehmer/innen ziehen Printpublikationen als Informationsquelle heran und ca. $51 \%$ nutzen hierfür auch Symposien. Vergleichsweise selten (nur von jeweils ca. einem Fünftel) werden Dienstleistungen von Unternehmensberater/innen sowie Förderprogramme zur Ausweitung des Wissenstandes über ausländische Märkte in Anspruch genommen.

\subsection{Teilnahmemotive an den Business Foren des WIFI}

Für rd. drei von vier Unternehmen war die Suche nach möglichen Kooperationspartnern ein Motiv, an den Businessforen teilzunehmen (siehe Grafik 9). An zweiter Stelle stand die Gewinnung besserer Marktkenntnisse über die ausländischen Märkte, die sich ca. $62 \%$ der Unternehmen von einem Besuch der Veranstaltung erwarteten.

Grafik 9 Gründe für eine positive Teilnahmeentscheidung an den Businessforen, Betriebe in Prozent *)

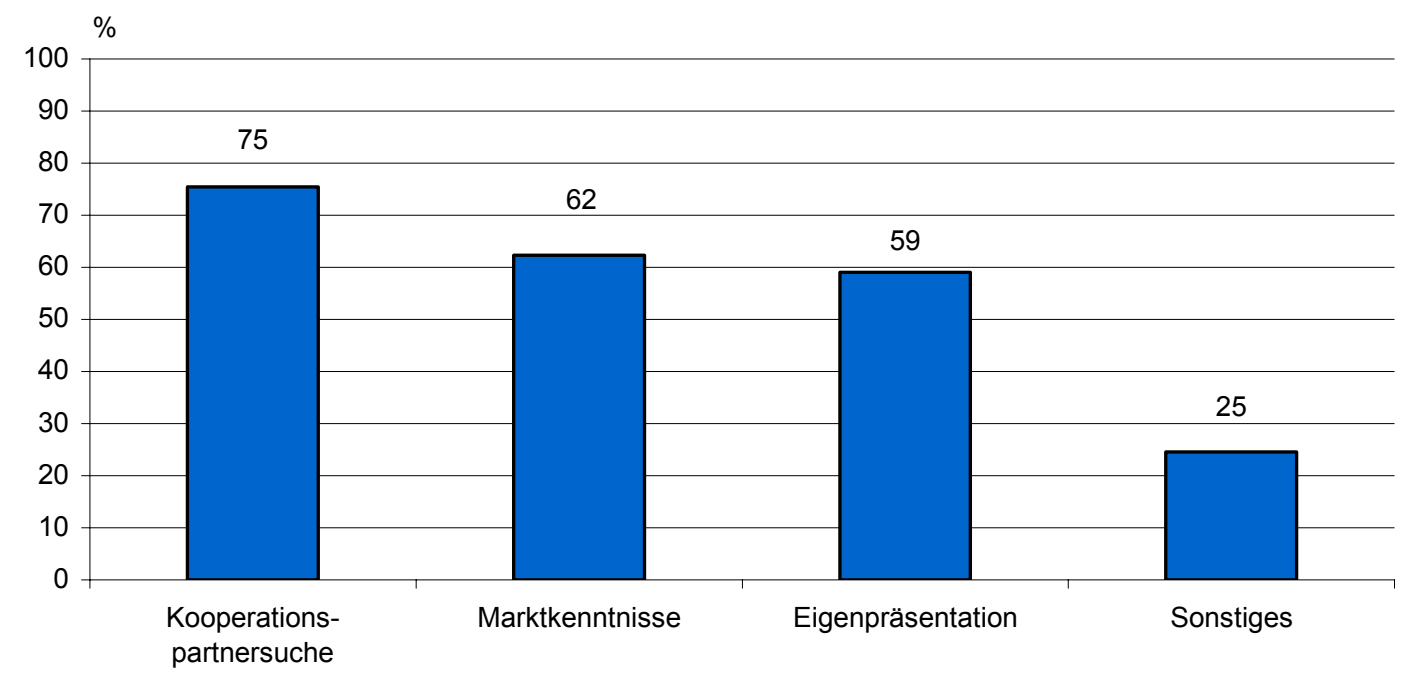

*) Mehrfachnennungen möglich

Quelle: KMU FORSCHUNG AUSTRIA, Unternehmensbefragung

Die hohe Bedeutung der Gewinnung besserer Marktkenntnisse überrascht in so fern, als dass die Betriebe ihren eigenen Kenntnisstand über osteuropäische Märkte sehr hoch einschätzen. Die Ergebnisse korrelieren jedoch mit den identifizierten Problemfeldern, da die Suche nach konkreten Informationen als zweitwichtigstes Problemfeld genannt wird (siehe hierzu auch Abschnitt 4.3.3). Viele für die Unternehmen relevante Informationen dürften nur schwer bis gar nicht erhältlich sein, sodass sich der hohe Kenntnisstand der Betriebe auf die (wohl nur teilweise positiven) Erfahrungen mit den bisherigen Informationsrecherchen beziehen dürfte und weniger darauf, dass alle benötigen Informationen tatsächlich auch zur Verfügung stehen.

Die Darstellung des eigenen Unternehmens im Rahmen des Businessforums war für ca. 59 \% der Betriebe wichtig, womit sich dieser Teilnahmeentscheidungsgrund an dritter Stelle wiederfindet. Sonstige Gründe spielten eine geringere Rolle. 


\subsection{Wirkungsbereiche der Business Foren}

\subsubsection{Kontakte zwischen österreichischen und osteuropäischen Unternehmen}

8 der 61 befragten Betriebe konnten auf dem Businessforum keine Kontakte knüpfen. Die restlichen 53 Unternehmen hatten während der Business Foren Kontakte mit ausländischen Betrieben, die bei 35 dieser 53 Unternehmen auch nach dem entsprechenden Forum aufrechterhalten wurden. Bei 13 Betrieben ist es schließlich zu Geschäftsabschlüssen gekommen (siehe Grafik 10).

Grafik 10 Kontaktstufen bzw. Intensität der Kooperation im Zuge der Businessforen

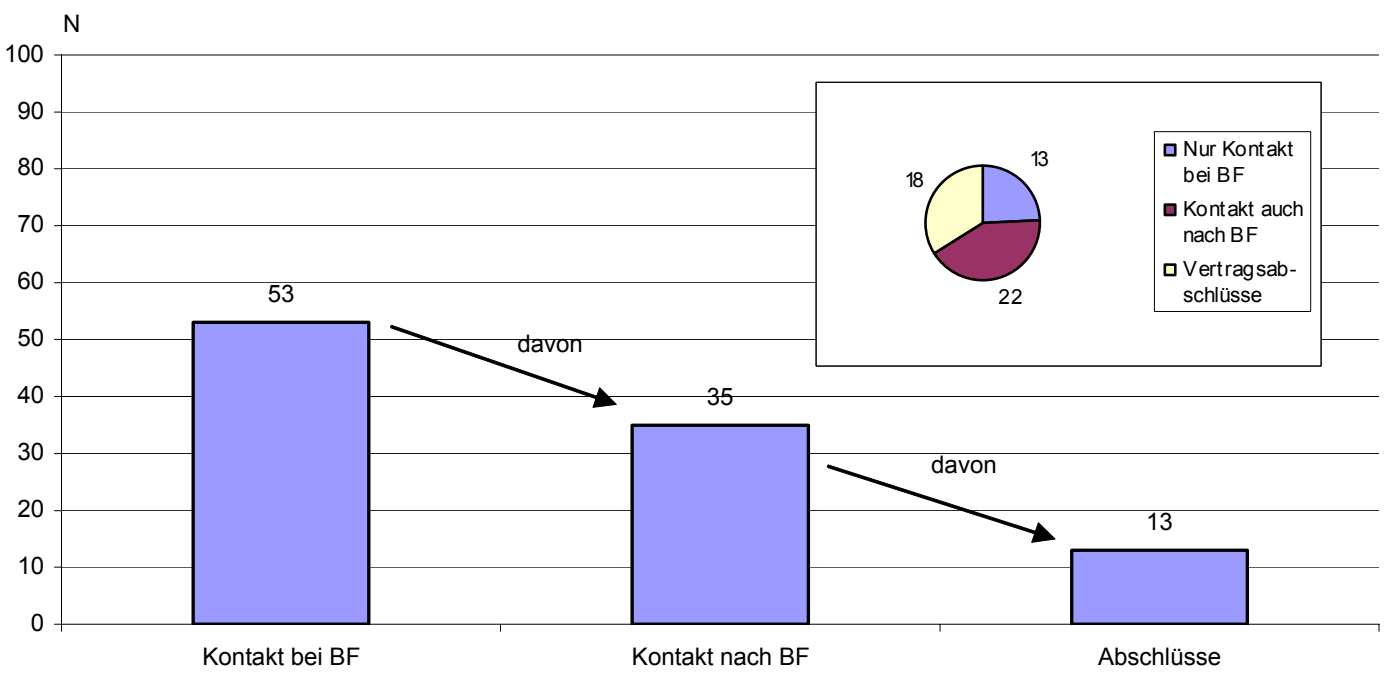

Legende: $\mathrm{BF}=$ Business Forum

Quelle: KMU FORSCHUNG AUSTRIA, Unternehmensbefragung

D. h., dass 22 Betriebe mehrfach mit ausländischen Betrieben in Kontakt getreten sind, (bislang) aber keine Vertragsabschlüsse tätigen konnten. Der Großteil (etwas mehr als zwei Drittel) dieser 22 Unternehmen hatte auf den Businessforen Kontakt zu potenziellen Kunden (siehe Grafik 11). Ca. 36 \% loteten Möglichkeiten für eine Zusammenarbeit im Produktionsbereich aus. Etwas weniger als ein Drittel der Unternehmen hatte Kontakt zu möglichen Lieferanten. Gespräche über F\&E-Kooperationen wurden lediglich von etwa $8 \%$ dieser 22 Unternehmen geführt. Kontakte für sonstige Kooperationsabsichten wurden von rd. $17 \%$ der österreichischen Betriebe geknüpft.

Es zeigt sich somit, dass in erster Linie Absatzmöglichkeiten für österreichische Produkte und Dienstleistungen in Osteuropa gesucht werden und erst in zweiter Linie über Zulieferungen bzw. Produktionskooperationen verhandelt wird. 
Grafik 11 Art der bei den Businessforen zustande gekommenen und anschließend verfestigten Kontakte, Betriebe in Prozent *)

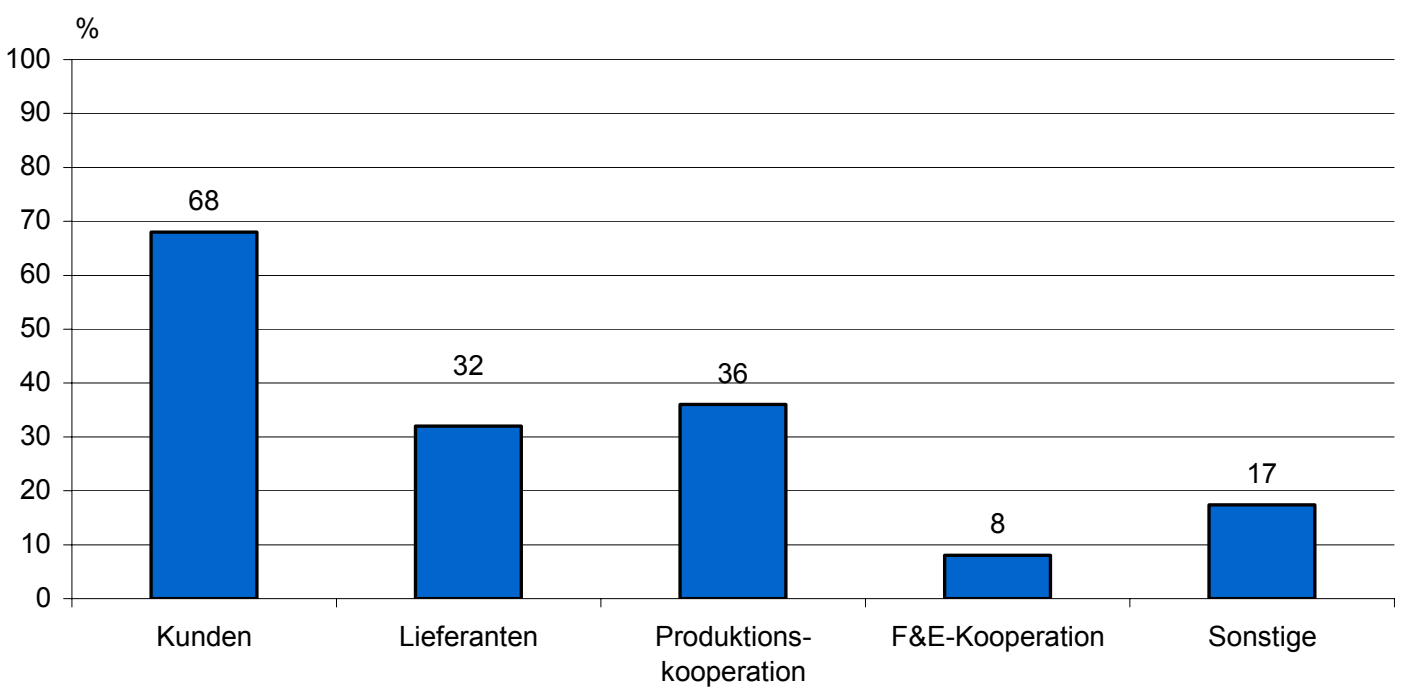

*) Mehrfachnennungen möglich

Quelle: KMU FORSCHUNG AUSTRIA, Unternehmensbefragung

Die auf den Businessforen zustande gekommenen Kontakte haben eine hohe Bedeutung für die österreichischen Unternehmen, auch wenn es zu keinen vertraglich geregelten Geschäftsabschlüssen kommt. Mehr als die Hälfte der Betriebe (rd. $56 \%$ ) bezeichnet ihre Kontakte als „wichtig“ für ihre Geschäftstätigkeit, weitere 28 \% immerhin als „eher wichtig“. Hingegen sehen lediglich $16 \%$ ihre Kontakte als „eher unwichtig“ oder gar „unwichtig“ an. Eine Mehrheit von ca. $60 \%$ der Unternehmen, die zwar mehrfach mit ausländischen Unternehmen in Kontakt getreten ist, aber keine verbindlichen Geschäftskontrakte abgeschlossen hat, plant auch langfristig mit osteuropäischen Unternehmen zusammenzuarbeiten. 
Grafik 12 Wichtigkeit der Kontakte für die Geschäftstätigkeit der österreichischen Unternehmen, Betriebe in Prozent

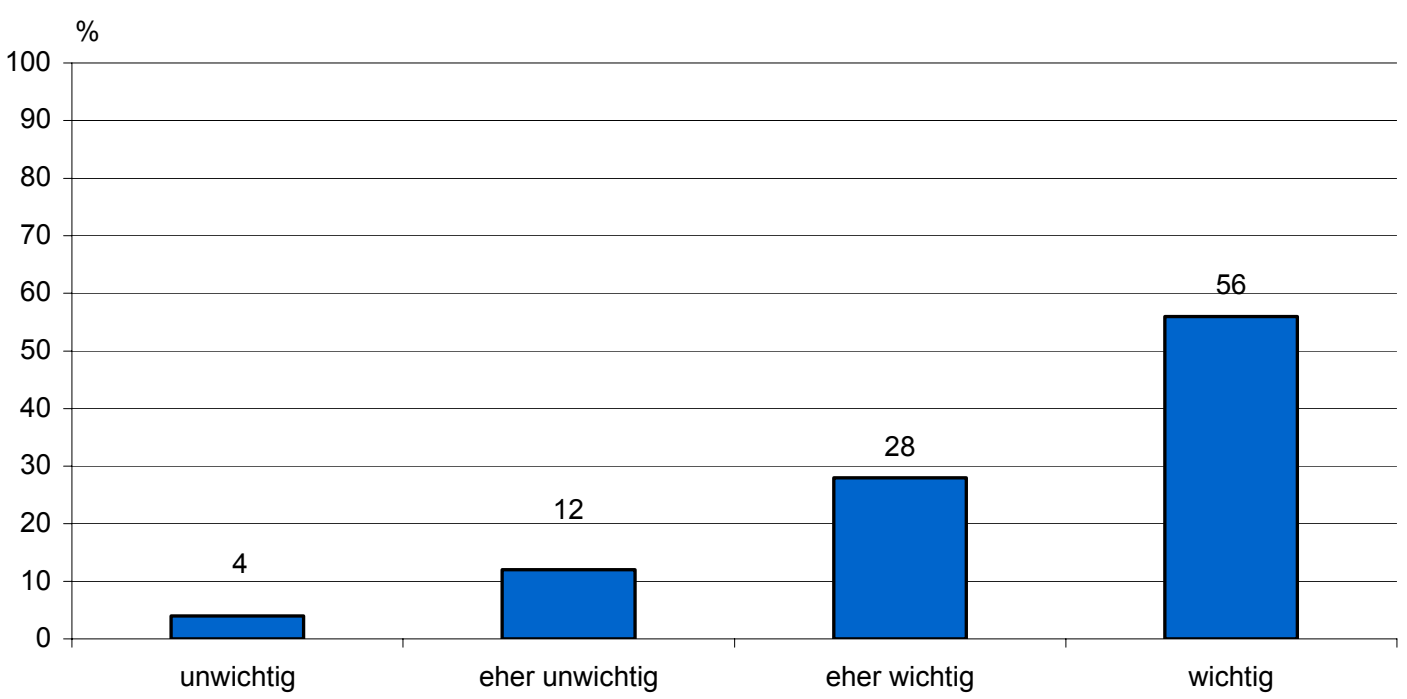

Quelle: KMU FORSCHUNG AUSTRIA, Unternehmensbefragung

\subsubsection{Vertragsabschlüsse zwischen österreichischen und osteuropäischen Unter- nehmen}

Bei 13 der 61 Unternehmen ist es in Folge des Besuchs von zumindest einem Businessforum zu Vertragsabschlüssen mit osteuropäischen Partnern gekommen. Die Mehrzahl dieser Verträge (insgesamt mehr als 40) sind Lieferverträge für Produkte und Dienstleistungen, vereinzelt wurden auch Kooperationsabkommen geschlossen, Unternehmen gegründet bzw. übernommen, Lizenzverträge abgeschlossen oder "sonstige Verträge“ unterzeichnet. Insgesamt haben die 13 Unternehmen 51 Verträge mit osteuropäischen Partnern abgeschlossen.

6 Unternehmen konnten das Volumen ihrer Geschäftsabschlüsse angeben oder zumindest schätzen. Begründet auf diesen Angaben liegt das gesamte Volumen der abgeschlossenen Verträge bei etwa $€ 17$ Mio, wobei $€ 15$ Mio hiervon auf das Kontraktvolumen eines einzelnen Betriebs (der BRAMAC GmbH, siehe auch Abschnitt 5.1) entfallen. 
Grafik 13 Art und Anzahl abgeschlossener Verträge österreichischer Unternehmen mit osteuropäischen Partnern

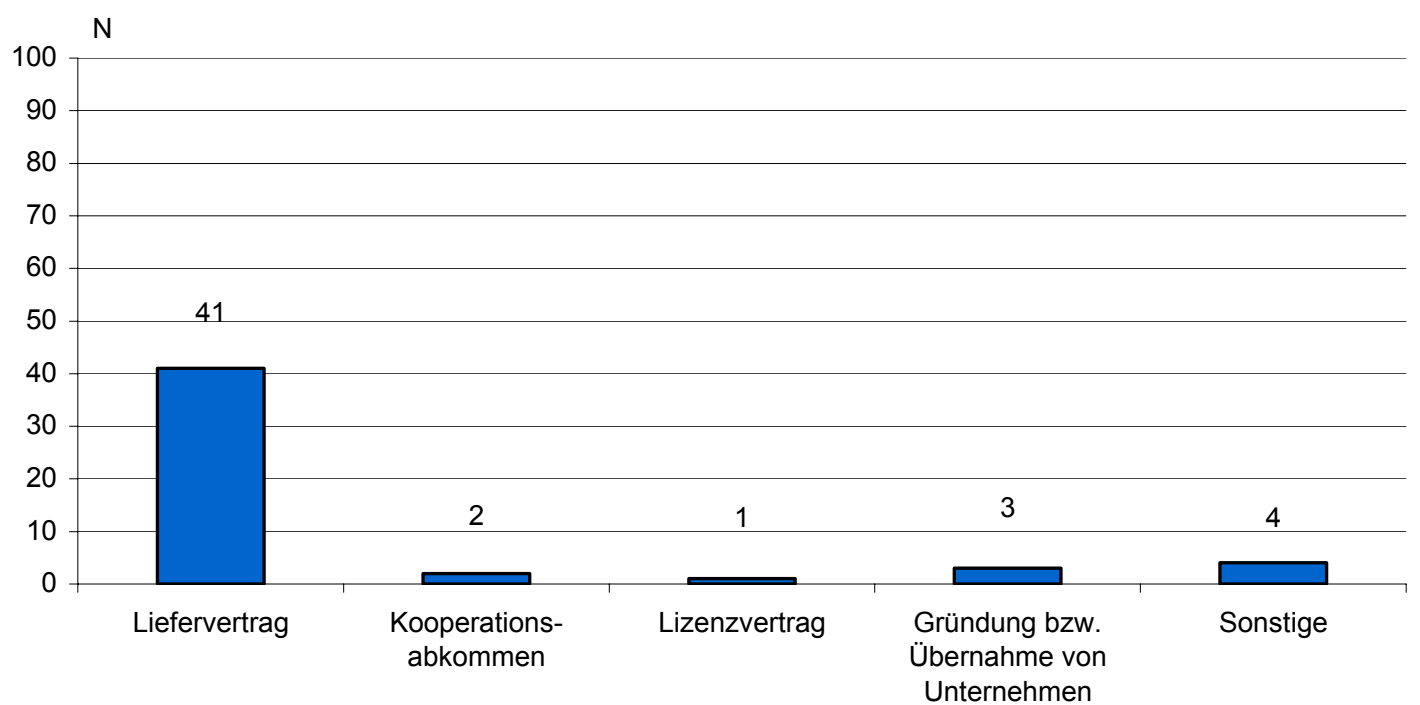

Quelle: KMU FORSCHUNG AUSTRIA, Unternehmensbefragung

Allerdings war nur für die Realisierung von ca. $€ 100.000$,- der $€ 17$ Mio die Teilnahme am Businessforum alleine entscheidend (reine Additionalitätseffekte). Es gilt aber auch festzuhalten, dass die reinen Mitnahmeeffekte lediglich $€ 250.000$,- ausmachen, d. h. dass Verträge im Ausmaß von $€ 250.000$,- nach Angabe der Unternehmen unabhängig von einer Teilnahme am Businessforum ohne zusätzlichen Aufwand und mit gleichem Partner zustande gekommen wären. Der restliche Teil von $€ 16,65$ Mio wäre ohne Besuch der Businessforen nur mit höherem Aufwand und/oder mit anderen Kooperationspartnern realisiert worden.

Grafik 14 Indirekte, durch Vertragsabschluss realisierte Auswirkungen, Betriebe absolut

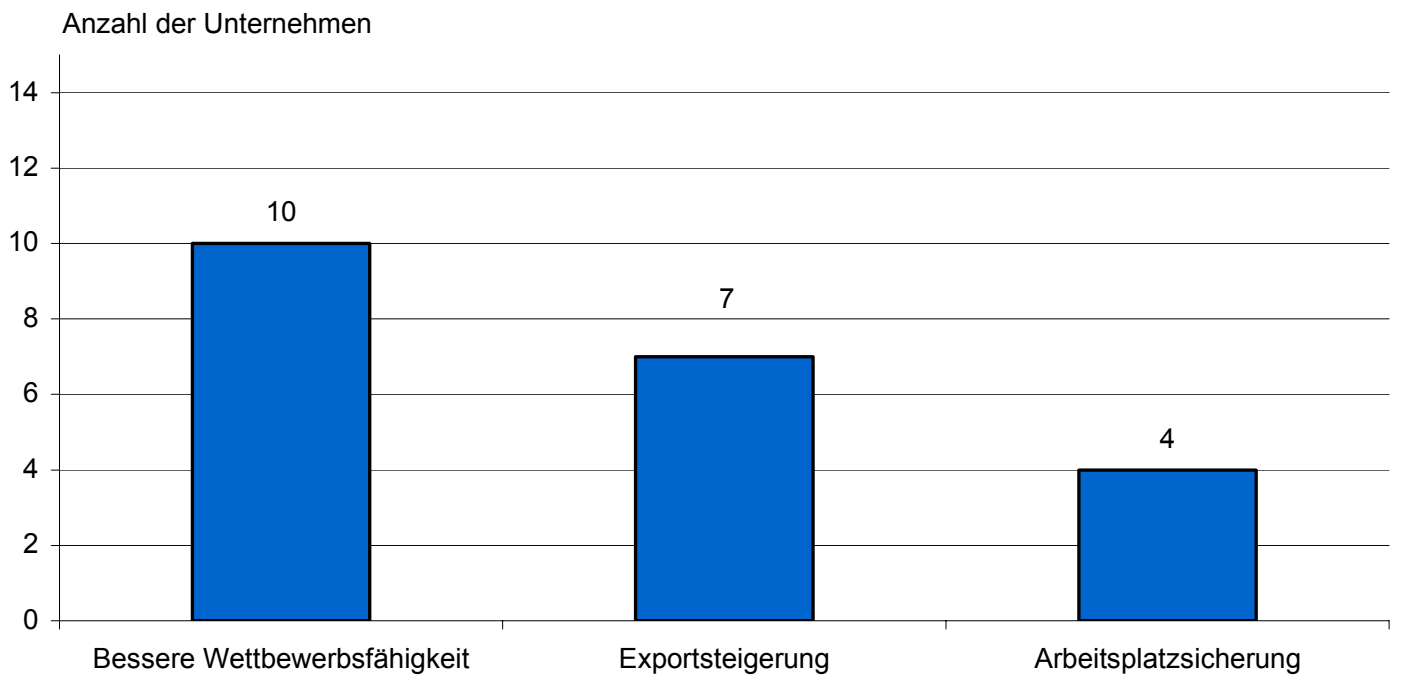

Quelle: KMU FORSCHUNG AUSTRIA, Unternehmensbefragung 
Neben den direkten monetären Auswirkungen wurden auch die Auswirkungen auf die Wettbewerbsfähigkeit, die Exporte und die Beschäftigung erhoben (siehe Grafik 14). Von den 13 Unternehmen, die in Folge der Businessforen Geschäftsverträge mit osteuropäischen Firmen abgeschlossen haben, gaben 10 an, dass die vertraglich realisierte(n) Kooperation(en) dazu beigetragen haben, die Wettbewerbsfähigkeit ihres Betriebs zu verbessern, 7 meldeten Exportsteigerungen und 4 gaben an, dass die Kooperation(en) Arbeitsplätze sichern halfen.

Für die Zukunft gehen 10 Unternehmen davon aus, dass über die bestehenden Verträge hinaus (z. B. im Rahmen von Folgeprojekten) mit den neuen Kooperationspartnern Zusatzumsätze lukriert werden. Auch aus diesem Grund wollen alle Unternehmen in dieser Gruppe in Zukunft mit osteuropäischen Betrieben weiter zusammenarbeiten.

\subsubsection{Nutzen und Problembereiche für alle beteiligten Unternehmen}

Die österreichischen Teilnehmer/innen der Businessforen bewerten den Nutzen der Businessforen vorsichtig positiv (siehe Grafik 15). Über alle beurteilten Nutzenfelder hinweg liegt der durchschnittliche Nutzen für die Betriebe in der Mitte der vorgegeben vierteiligen Nutzenskala (Durchschnittswert: 2,4). Die Differenzierung nach Nutzenfeldern zeigt ein sehr homogenes Bild mit nur geringen Abweichungen vom Gesamtdurchschnittswert: Die Bewertungen liegen im Durchschnitt zwischen 2,6 (höhere Bekanntheit des eigenen Unternehmens) und 2,2 (bessere Kenntnisse bezüglich ausländischer Geschäftspraktiken).

Grafik 15 Nutzen der Businessforen für die österreichischen Betriebe, Durchschnittswerte*)

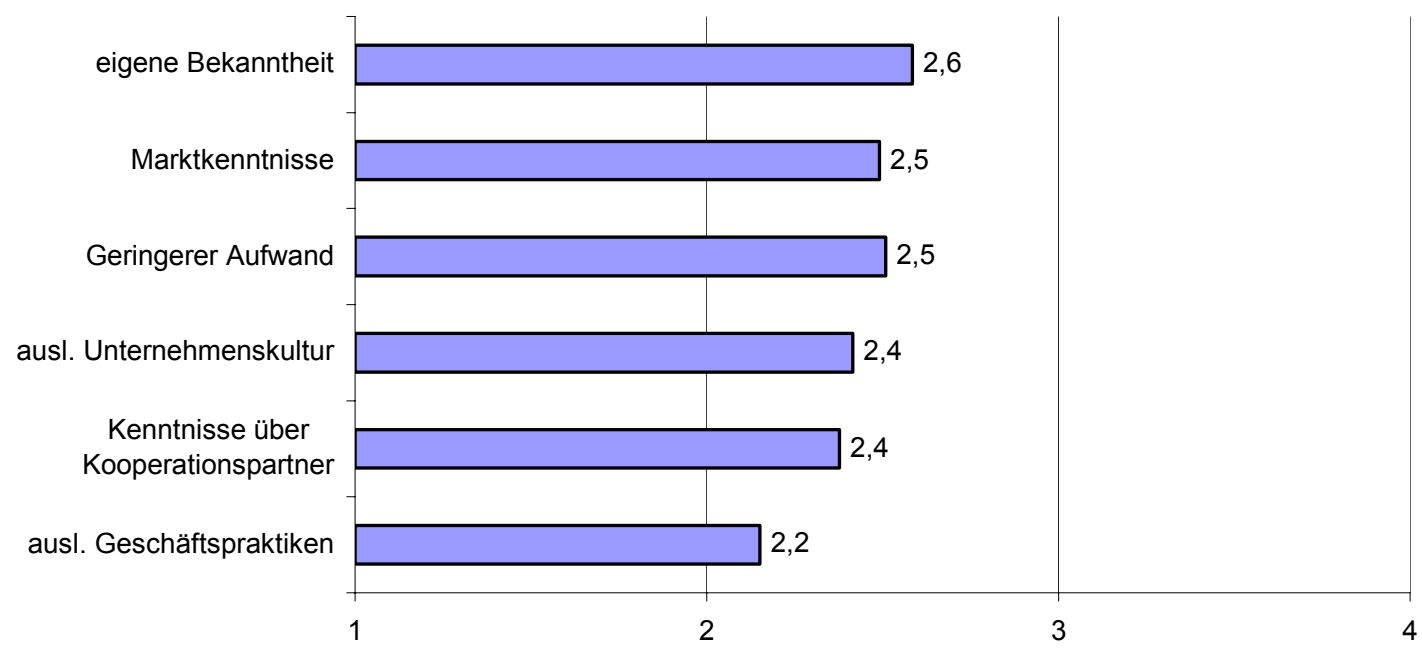

*) auf einer Skala von 1=kein Nutzen, 2=geringer Nutzen, 3=hoher Nutzen bis 4= sehr hoher Nutzen Quelle: KMU FORSCHUNG AUSTRIA, Unternehmensbefragung

Die durchwegs vorhandene Zufriedenheit der österreichischen Manager/innen mit den Businessforen drückt sich auch in einem hohen Anteil von fast $95 \%$ an Unternehmen aus, die wieder an einer derartigen Veranstaltung teilnehmen würden. In zahlreichen Interviews konnte festgestellt werden, dass die Ausbildungslehrgänge nach Ansicht der Betriebe prinzipiell einen wichtigen Beitrag zur Bearbeitung und zum Abbau von Problemfeldern in der Zusammenarbeit mit osteuropäischen Unternehmen liefern können - speziell in Bezug auf die konzentrierte und aufwandsreduzierte Beschaffung von Informationen über potenzielle Kooperationspartner und ausländische Märkte sowie die Vermittlung von westlichem Managementwissen an osteuropäische Betriebe. 
Der wichtigste Problembereich für die österreichischen Teilnehmer/innen an den Businessforen ist - im Hinblick auf eine etwaige Zusammenarbeit mit osteuropäischen Unternehmen - das häufig fehlende Know-How der ausländischen Betriebe (siehe Grafik 16). Etwa sieben von zehn Teilnehmer/innen bemängelten in den Gesprächen Faktoren wie "zu produktionslastige“ Managementmethoden, eingeschränkte Marketingkenntnisse oder wenig flexible, bürokratische Organisationsstrukturen auf Seiten der ausländischen Partner (siehe hierzu auch Abschnitt 5). Obwohl diesbezüglich schon enorme Fortschritte in Osteuropa gemacht wurden, zeigt sich demnach ein deutlicher Bedarf an entsprechenden Schulungsmaßnahmen und Ausbildungsprogrammen.

Erstaunlich ist auf den ersten Blick auch der relativ hohe Anteil an Unternehmen (ca. $66 \%$ ), die Schwierigkeiten bei der Beschaffung konkreter Informationen haben. Immerhin geben die meisten Unternehmen an, „sehr gut“ oder "gut“ über die ausländischen Märkte informiert zu sein und können auf einen reichen Erfahrungsschatz in Bezug auf ihre Geschäftstätigkeit in Osteuropa zurückgreifen (siehe Abschnitt 4.1).

Zusammengenommen deutet dies darauf hin, dass viele wichtige Informationen entweder gar nicht verfügbar oder nur sehr schwer zugänglich sind, sodass eine intensive und aufwendige Recherchearbeit zur Deckung zumindest des grundsätzlichsten Informationsbedarfs unerlässlich ist (siehe auch Abschnitt 4.2).

Beinahe jedes zweite Unternehmen (rd. $49 \%$ ) sieht des Weiteren fehlendes Vertrauen - ein klassisches Problemfeld für KMU im Hinblick auf das Kooperationsverhalten - in ausländische Geschäftspartner als Problembereich an. Für rd. $44 \%$ ist außerdem häufig das Risiko eines Auslandsengagements zu hoch.

Kulturelle Unterschiede können für ca. ein Drittel der Betriebe die Zusammenarbeit mit osteuropäischen Unternehmen erschweren und etwa ein Viertel sieht sprachliche Verständigungsprobleme. Die relativ niedrige Bedeutung der beiden letzten Problemfelder könnte auf eine Reihe von Faktoren zurückzuführen sein. Zum einen dürfte der Umgang mit der osteuropäischen Mentalität österreichischen Unternehmen leichter fallen als anderen Nationen, da noch gemeinsame kulturelle Wurzeln aus der Monarchiezeit vorhanden sind. Zum anderen haben sich aber auch die Fremdsprachenkenntnisse auf beiden Seiten verbessert. 
Grafik 16 Identifizierte Problembereiche bei der Zusammenarbeit mit osteuropäischen Unternehmen, Betriebe in Prozent *)

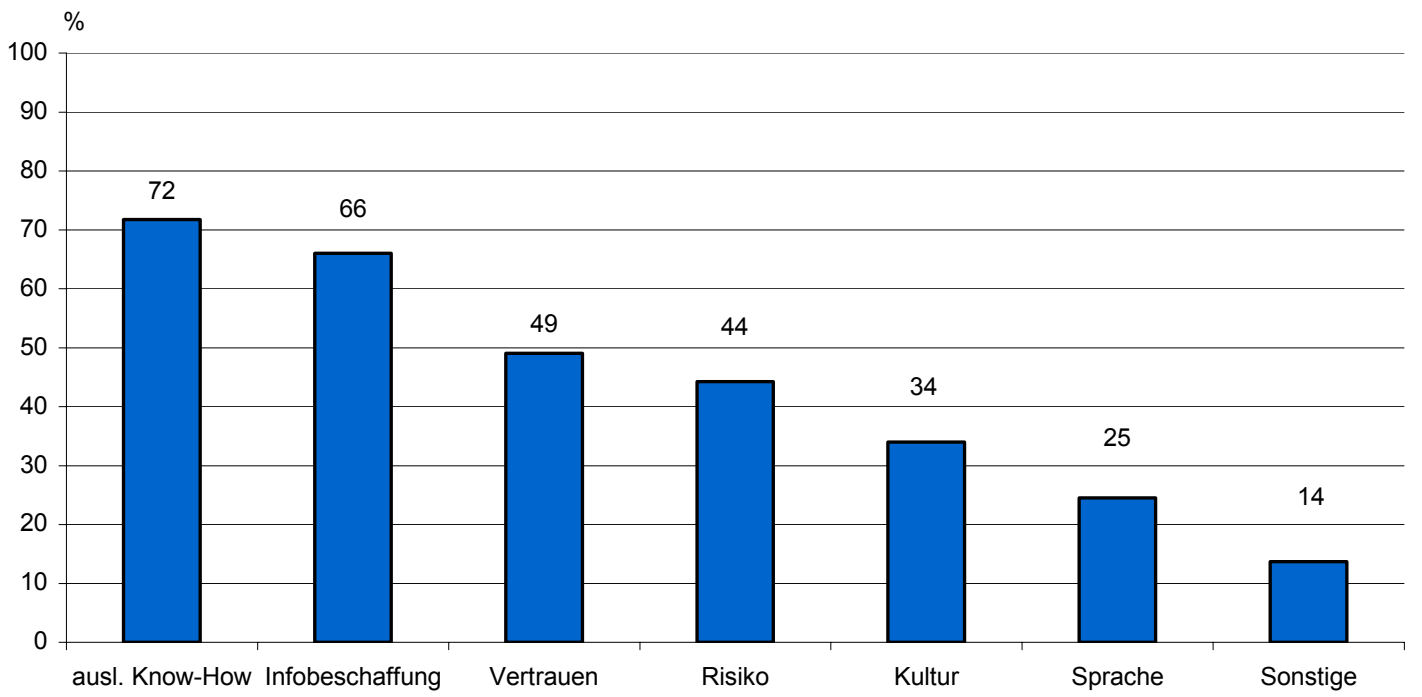

*) Mehrfachnennungen möglich

Quelle: KMU FORSCHUNG AUSTRIA, Unternehmensbefragung

Differenziert nach Unternehmensgröße zeigt sich, dass die Problembereiche von Betrieben mit mehr als 250 Beschäftigten vom allgemeinen Bild etwas abweichen. Große Betriebe sehen sich im Wesentlichen mit zwei Problemfeldern konfrontiert: Schwierigkeiten bei der Beschaffung konkreter Informationen und - in geringerem Ausmaß - sprachlichen Verständigungsproblemen.

Über die vorgegeben Problemfelder bei der Geschäftstätigkeit in Osteuropa hinaus wurden in den Gesprächen mit den Unternehmen (siehe hierzu auch Abschnitt 5) und Programmverantwortlichen auch mögliche Problembereiche bei der Implementierung des Programms identifiziert. So erweist sich die Organisation der Businessforen durch die AWO zuweilen als schwierig. Da die Organisation dieser Veranstaltungen nicht den Kerntätigkeiten der AWO zuzurechnen ist (im Gegensatz zur Durchführung der Lehrgangsprogramme durch die IKH) und die für die Durchführung verantwortlichen Personen häufig wechseln, dürfte auch die Qualität der Organisation der Businessforen variieren. Dies betrifft weniger den Ablauf der Businessforen vor Ort als die Auswahl der teilnehmenden ausländischen und österreichischen Betriebe sowie die Zusammenarbeit mit dem IKH.

Hinsichtlich der eingeladenen ausländischen Betriebe wird vielfach kritisiert, dass diese nur unzureichend darüber informiert sind, was sie konkret von einem Businessforum erwarten können. Im Speziellen herrscht der Eindruck vor, dass manche osteuropäischen Betriebe davon ausgehen dass eine höhere Anzahl österreichischer Unternehmen zwecks schneller Liefervertragsabschlüsse auf den Businessforen „Schlange stehen“ würde. Andererseits gibt es aber auch Unternehmen, die zwar an einem Businessforum teilnehmen (z.B. um Österreich als Land ein wenig kennen zulernen) wohl aber keine Kooperationsabsichten verfolgen. Schließlich scheint es auch häufig vorzukommen, dass osteuropäische Betriebe ihre Erwartungen an mögliche österreichische Partner nur unzureichend formulieren und lediglich sehr allgemeine Beschreibungen ihrer Geschäftsfelder liefern. Damit wird es für potenzielle österreichische Teilnehmer/innen sehr schwer abzuschätzen, ob tatsächlich für sie interessante Geschäftspartner bei den Businessforen anwesend sind. Insgesamt wird ersichtlich, dass der Selektionsprozess der ausländischen Unternehmen vor Ort Optimierungspotenzial aufweist und vorhandene Erwartungen u.ä. vielfach genauer erhoben werden müssten. 
Eine Reihe von Verbesserungsmöglichkeiten zeigen sich auch bei der Ansprache inländischer Betriebe. Der schriftlichen Aussendung folgen meist keine weiteren Schritte um die Unternehmen zur Teilnahme zu motivieren. Ein vermehrtes Nachfassen bzw. Nachtelefonieren könnte zu einer höheren Teilnahmefrequenz führen. Als wenig vorteilhaft erweist sich zudem die zentrale SQL Adressdatenbank. Diese ist einerseits relativ schwer zu bedienen. Andererseits sind manche Datenbankfelder schlicht unterdimensioniert und erlauben es daher nicht, umfangreichere Angaben der Unternehmen über ihre Erfahrungen und Erwartungen abzuspeichern. Für Firmen, denen das Konzept des Businessforums neu ist, fehlen schließlich bei der Aussendung oft ergänzende Informationen, z.B. über die ausländischen Märkte etc. 


\section{Good Practice Beispiele}

Im Folgenden werden drei Unternehmen vorgestellt, die aus den Businessforen überdurchschnittlichen Nutzen gezogen haben. Ihre positiven Erfahrungen qualifizieren diese Betriebe als "Good Practice“ Beispiele und illustrieren, wie die Businessforen des WIFI Unternehmen bei der Geschäftsanbahnung in Osteuropa helfen können. Gleichzeitig liefern die durch diese Unternehmen identifizierten Problembereiche wichtige Anhaltspunkte für mögliche Optimierungen bei Programminhalten und -abläufen.

\subsection{Der Dachstein- und Dachsystemhersteller BRAMAC}

Die „BRAMAC Dachsteinwerke International GmbH“, ein Produzent von Dachsteinen und Dachsystemen, wurde 1966 gegründet und hat seine Zentrale in Pöchlarn in Niederösterreich. Das Unternehmen gehört zu jeweils $50 \%$ der Wienerberger AG und der Lafarge Roofing und beschäftigt zurzeit etwa 260 Mitarbeiter/innen.

\section{Grafik $17 \quad$ Standorte der BRAMAC GmbH, Stand 11.12.2003}

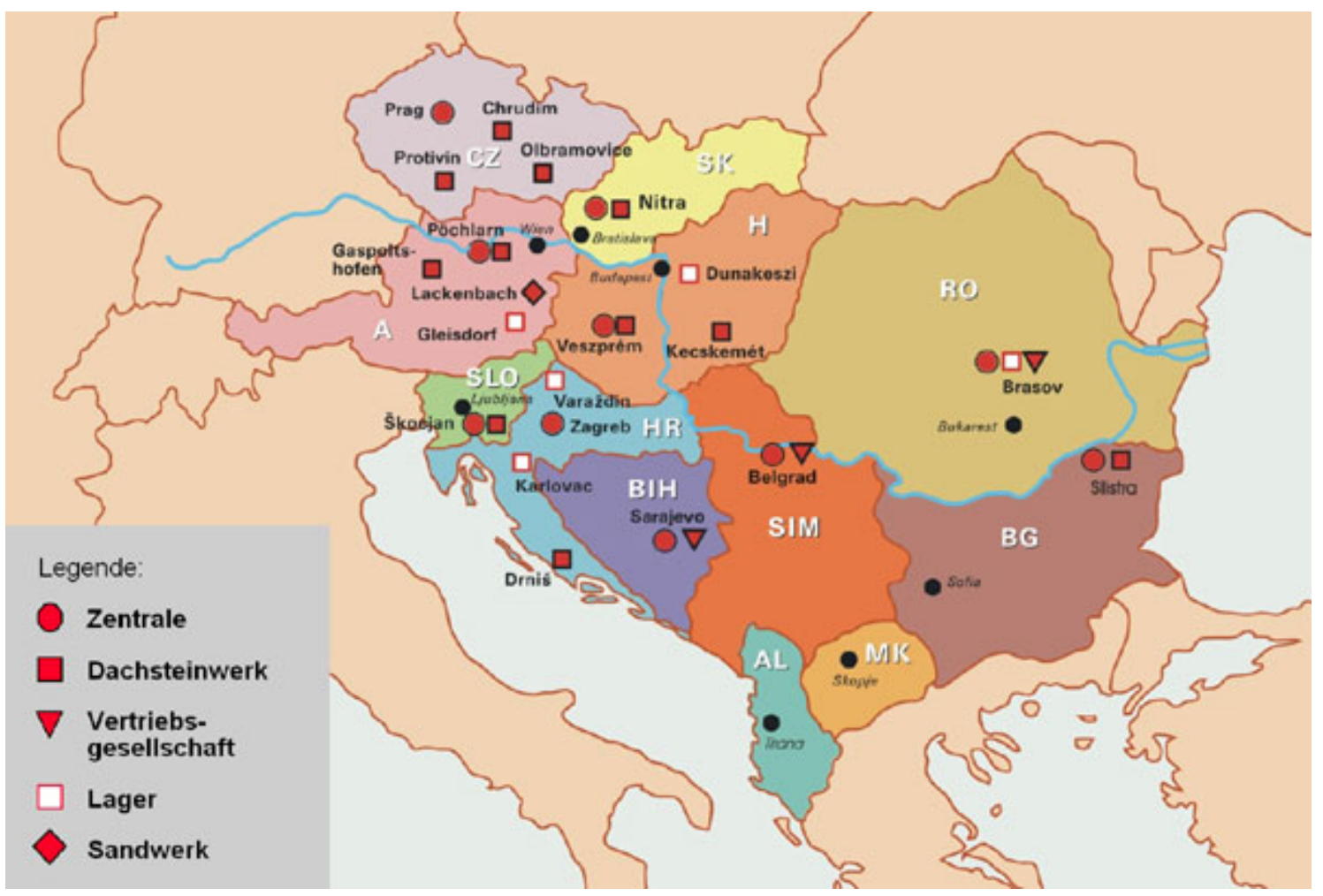

Quelle: Homepage der BRAMC Gmbh, http://www.bramac.com

Schon relativ früh wurde das Marktpotenzial in Osteuropa erkannt und bereits 1984 ein erstes Joint Venture Abkommen mit einem ungarischen Betrieb abgeschlossen. Mit dem Fall des eisernen Vorhangs beschleunigte sich die Expansion in Osteuropa. Die Expansionsstrategie sieht für jedes Land vor, zuerst die eigenen Produkte direkt an ausländische Abnehmer zu verkaufen, dann einzelne Werke zu akquirieren und eine Vertriebsgesellschaft zu gründen. Hat der Marktanteil schließlich einen entsprechenden Wert erreicht erfolgen weitergehende Investitionen und die Gründung einer eigenen Niederlassung bzw. Zentrale. Derzeit ist die BRAMAC in 11 osteuropäischen Ländern aktiv (siehe Grafik 17). 
Die BRAMAC AG war in den letzten Jahren bei mindestens 8 Businessforen mit rumänischen, bulgarischen, bosnischen und serbischen Teilnehmer/innen anwesend. Der erste Vertrag, der durch eine Teilnahme an einem Businessforum zustande kam, beinhaltete eine Lieferung von Dachsystemen an die serbische Firma Constanza und wies ein Volumen von ca. $€ 10.000$,- auf. Dieses relativ niedrige Vertragsvolumen ist It. BRAMAC typisch für eine beginnende Expansion in ein neues Land. Der Aufbau von strategischen Partnerschaften, das Kennenlernen der Marktbesonderheiten und die schrittweise Erhöhung der Marktanteile sind in dieser Phase wichtiger als die Erzielung hoher Umsätze.

Mittlerweile machen die durch weitere Kontakte bei den Businessforen entstandenen Verträge ein Volumen von insgesamt etwa $€ 15$ Mio aus. Damit ist die BRAMAC GmbH jenes Unternehmen, das in monetärer Hinsicht am meisten von den Businessforen profitiert hat.

Die BRAMAC schätzt an den Businessforen vor allem zwei Aspekte:

- Zum einen die sich ergebende Aufwandsverringerung, da eine sehr hohe Zahl an möglichen Geschäftspartner/innen an einem Ort versammelt ist. Die Tatsache, dass die Foren in Österreich stattfinden reduziert zudem den Reiseaufwand, der in Osteuropa nicht unerheblich ist.

- Zum anderen die Möglichkeit auch branchenübergreifend Erfahrungen auszutauschen.

Die Businessforen fingieren für die BRAMAC meist als Impulsgeber, bei denen ersten Kontakten und kleineren Vertragsabschlüssen weitergehende Kontakte und höhervolumige Aufträge folgen.

Schwächen sieht das niederösterreichische Unternehmen vor allem bei der Anwendung modernen betriebswirtschaftlichen Know-Hows durch die osteuropäischen Betriebe. Dementsprechend positiv werden auch die Kursprogramme des WIFI in Osteuropa bewertet. Die BRAMAC empfiehlt, sich hier vor allem der Bereiche Marketing und Vertrieb verstärkt anzunehmen. Vielfach würde Marketing noch mit Werbung gleichgesetzt werden.

\subsection{Der Batterienerzeuger BÄREN BATTERIE}

Die BÄREN BATTERIE in Feistritz in Kärnten liefert Batterien für verschiedenste Einsatzwecke, z.B. Starterbatterien, Spezialakkumulatoren oder Notstromaggregate. Das Unternehmen ist eine Tochtergesellschaft der italienischen FIAMM Gruppe, die weltweit etwa 3.400 Mitarbeiter/innen beschäftigt. In Kärnten sind ca. 75 Personen tätig.

Neben der Bearbeitung des österreichischen Markts ist die BÄREN BATTERIE für den Großteil des Osteuropageschäfts des FIAMM Konzern zuständig. Dies begründet sich It. BÄREN BATTERIE mit den österreichischen Mitarbeiter/innen zugestandenen Kenntnissen osteuropäischer Märkte bzw. deren besonderen Verständnis für die osteuropäische Kultur.

Das Unternehmen hat an einem Businessforum mit rumänischen Betrieben im Jahr 2001 teilgenommen und konnte in der Folge im Jahr 2002 einen Liefervertrag mit einem rumänischen Telekommunikationsanbieter abschließen (realisiertes Vertragsvolumen: $€ 35.000,-)$. Außerdem war die BÄREN BATTERIE bei einem bulgarischen Businessforum anwesend, wobei es bislang aber noch zu keinen Vertragsabschlüssen gekommen ist.

Ein wesentlicher Problembereich, mit dem sich der Kärntner Betrieb in Osteuropa konfrontiert sieht, sind überdurchschnittlich lange Instanzenwege, die sich aus veralteten hierarchisch-bürokratischen Organisationsstrukturen ergeben. Da die Kunden des Batterienherstellers meist größere Unternehmen sind, dauert es entsprechend lange bis - nach etlichen Rückfragen und Weiterleitungen - tatsächlich mit einem entscheidungsbefugten Mitglied des Managements verhandelt werden kann. 
Hier sieht die BÄREN BATTERIE den größten Vorteil der Businessforen: Führungspersonen können unter Umgehung sämtlicher niedrigerer Organisationsebenen direkt angesprochen werden. Hinzu kommt, dass durch die Teilnahme der osteuropäischen Manager auch eine Eigeninitiative sichtbar wird, d.h. dass der osteuropäische Betrieb aktiv nach Kooperationsmöglichkeiten sucht, was eine positive Kontaktaufnahme wesentlich erleichtert. Schließlich ist auch der verminderte Aufwand (geringere Reisen, höhere Anzahl potenzieller Kooperationspartner an einem Ort) ein wesentlicher Aspekt für die Teilnahme an den Businessforen.

Wichtig ist jedoch It. BÄREN BATTERIE, dass im Vorfeld der Businessforen genau selektiert wird und tatsächlich nur jene Unternehmen eingeladen werden, die explizit ein Interesse an Kooperationen mit österreichischen Unternehmen haben bzw. über ein für österreichische Betriebe interessantes Unternehmensprofil (z.B. hinsichtlich des Produktportfolios, einer entsprechenden Betriebsgröße etc.) verfügen.

\subsection{Die ATB AUSTRIA Antriebstechnik}

Bei der Firma ATB Antriebstechnik handelt es sich um ein Spin-Off der Firma Bauknecht, das sich seit der Gründung im Jahr 1988 auf die Produktion und den Vertrieb von Elektromotoren und Antriebssystemen spezialisiert hat. In Österreich waren bei der ATB im Jahr 2002 rd. 645 Mitarbeiter/innen beschäftigt, der Jahresumsatz lag bei rd.€81 Mio, wovon etwa $86 \%$ exportiert wurden. Die ATB verfügt sowohl über eigene Niederlassungen im Ausland als auch über Vertriebspartner.

Die ersten Kontakte mit den Businessforen hatte das Unternehmen im Jahr 1999. Nach mehrfachen Verhandlungen sowie einer Messeteilnahme in Sarajewo konnte schließlich ein Liefervertrag über Elektromotoren mit einer Firma aus Belgrad abgeschlossen werden. Seitdem war die ATB bei jeweils einem rumänischen, bulgarischen und bosnischen sowie bei drei serbischen Businessforen anwesend. Das Volumen der durch die Businessforen initiierten Verträge lag insgesamt bei etwa $€ 500.000$,-, wobei aber ein nicht näher zu bestimmender Teil auch ohne die Businessforen (mit höherem Aufwand) realisiert hätte werden können. Wichtiger Pluspunkt der Businessforen ist It. ATB die Möglichkeit des Kennenlernens fremder Unternehmenskulturen.

Die Erfahrungen der ATB zeigen, dass Engagements in Osteuropa meist viel Mühe bereiten und Geduld erfordern. Firmen, die Anfragen unbeantwortet lassen, aber auch Sprachprobleme gehören zur Tagesordnung. Dazu kommt eine meist nicht ausreichend entwickelte Infrastruktur, die das Reisen deutlich erschwert. Selbst wenn erste Verträge geschlossen wurden, ist es It. ATB immer noch diffizil, Marktanteile auszubauen und weitere Verträge zu realisieren. Ein gewisser Durchhaltewille ist unerlässlich.

Für die Businessforen ist es It. ATB notwendig, dass die osteuropäischen Teilnehmer/innen genau darüber informiert werden, was sie bei den Businessforen erwartet bzw. erwarten könnte. Zum Teil besteht der Eindruck, dass die Erwartungshaltungen bei einigen osteuropäischen Unternehmen deutlich überzogen sind und ungenaue Vorstellungen darüber herrschen, wie Geschäfte im Ausland abgeschlossen werden. 